\title{
Survey of Department of Defense Facilities with Low NOx Burners
}

\author{
Peter Herz \\ George Powers \\ Douglas Uthus \\ Steven Aylor \\ Michael K. Brewer
}

This study conducted a survey regarding recent experience with Low NOx Burners (LNBs) at Department of Defense facilities known to be operating or installing low nitrogen oxidesemitting burners on boilers. The data collected did not conclusively show that LNBs are chronically problematic, but a majority of the LNB-equipped boilers recorded in the data base reported problems with operation, installation, or commissioning. The most frequently reported problems were the failure of the units to reduce NOx emissions to the level promised and flame instability caused by a variety of factors. 


\section{Executive Summary}

Between October 1998 and February 1999, Technology \& Management Services, Inc. conducted a survey for the U.S. Army Construction Engineering Research Laboratory (CERL) regarding recent experience with Low NOx Burners (LNBs). The survey involved contacting 50 Department of Defense facilities operating or installing low nitrogen oxides-emitting burners on boilers and acquiring information on experience associated with the burners. Of the 28 facilities that responded to the questionnaire, only 13 reported operating LNBS and provided useful information. The data collected did not conclusively show that LNBs are chronically problematic, but a majority (10 of 16) of the LNB-equipped boilers recorded in the data base reported problems with operation, installation, or commissioning. The most frequently reported problems were the failure of the units to reduce $\mathrm{NOx}$ emissions to the level promised and flame instability caused by a variety of factors. Numerous innovative solutions to these problems were reported in the survey, but overall, the LNBs seem to be a source of significant frustration. 


\section{Foreword}

This study was conducted for U.S. Army Corps of Engineers (USACE) under Project 4A162784AT45, "Energy and Energy Conservation;" Work Unit UL-XC7, "Advanced Energy Supply Technology." The technical monitor was J ohn Lanzarone, CEMP-ET.

The work was performed by the Energy Branch (CF-E), of the Facilities Division (CF), U.S. Army Construction Engineering Research Laboratory (CERL). Survey data was gathered by Technology \& Management Services, Inc., Gaithersburg, MD, under contract DACA-98-D-0007. Peter Herz, George Powers, Douglas Uthus, and Steven Aylor are associated with Technology \& Management Services, Inc. The CERL principal investigator was Michael K. Brewer. Larry M. Windingland is Chief, CECER-CF-E and Dr. L. Michael Golish is Chief, CECERCF. The CERL technical editor was William J. Wolfe, Information Technology Laboratory.

The Director of CERL is Dr. Michael J . O'Connor. 


\section{Contents}

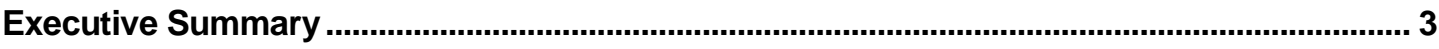

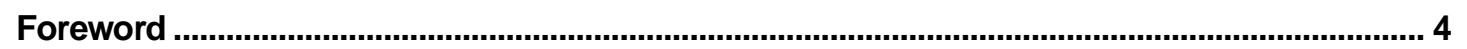

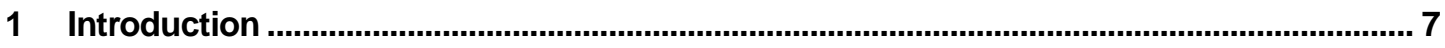

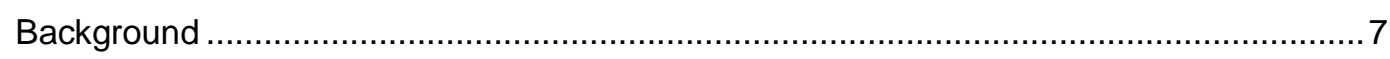

Objectives

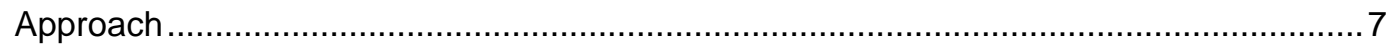

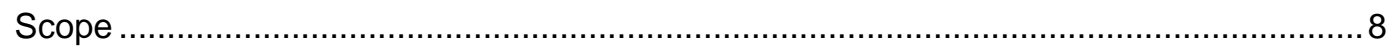

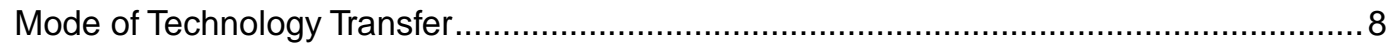

2 Facility Identification $\ldots$

3 Survey Questionnaire and Data Base Development.......................................................11

Questionnaire Development..................................................................................

Questionnaire Testing and Distribution....................................................................11

4 Results........................................................................................ 13

Level of Response

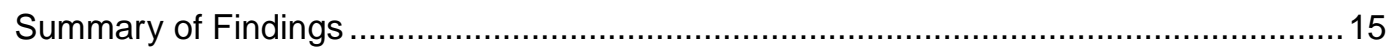

5 Conclusions and Recommendations ............................................................................ 18

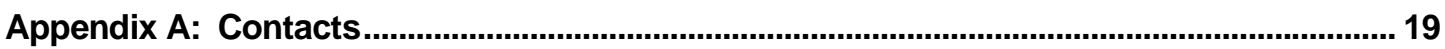

Appendix B: Questionnaire

Appendix C: Survey Results.............................................................................................. 27

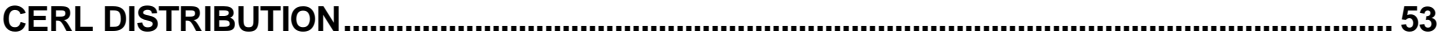

REPORT DOCUMENTATION PAGE ................................................................................... 54 


\section{Introduction}

\section{Background}

The U.S. Army Construction Engineering Research Laboratory (CERL) contracted with Technology \& Management Services, Inc. (TMS) to conduct a survey of Department of Defense (DOD) facilities that have installed new burners to control emissions of nitrogen oxides (NOx) on gas- and oil-fired boilers. The low NOx burners (LNBs) are necessary to meet increasingly stringent air quality limits under the Clean Air Act Amendments of 1990 (CAAA). Operation with the LNBs, however, has raised concerns regarding the safety and performance of DOD industrial-sized boilers. Problems with flame control during LNB operation have been reported. There has also been a concern that manufacturers are not following the suggestions regarding LNBs contained in the non-binding appendix to the National Fire Protection Association (NFPA) Standard 8501, Standard for Single Burner Operation, 1997 Edition. These suggestions include relocating flame scanners and retesting operating margins after LNB retrofit.

\section{Objectives}

The objective of this study was to document installation, commissioning, and operational problems encountered with LNBs.

\section{Approach}

The method used to conduct the survey was outlined in the Research Plan TMS submitted to CERL on 30 October 1998. Facilities using LNB technology were identified. A questionnaire and data base were developed and tested on five initial facilities. The questionnaire was then sent to the rest of the facilities, and responses were collected. Data contained in the responses were then entered into the data base and analyzed. 


\section{Scope}

This survey was sent to DOD military installations known to have recent experience with LNBs. Results of the survey, and condusions drawn from the survey are intended to pertain to these applications.

\section{Mode of Technology Transfer}

This report and the raw survey data collected will be made available to aid in future DOD and manufacturer decisions about the design, application, and safety of LNB technology, through CERL's world-wide web (WWW) URL:

http://www.cecer.army.mil. 


\section{Facility Identification}

CERL identified 32 U.S. Army and other facilities to contact for the survey, as well as contact information for persons or organizations with information on U.S. Air Force and U.S. Navy installations operating LNBs. The 32 installations identified by CERL were:

$\begin{array}{lll}\text { Aberdeen Proving Ground } & \text { Fort Carson } & \text { Fort Hood } \\ \text { Carlisle Barracks } & \text { Fort Dix } & \text { Fort Jackson } \\ \text { Fort Belvoir } & \text { Fort Drum } & \text { Fort Knox } \\ \text { Fort Benning } & \text { Fort Eustis } & \text { Fort Leonard Wood } \\ \text { Fort Bragg } & \text { Fort George G. Meade } & \text { Fort Lewis } \\ \text { Fort Campbell } & \text { Fort Gordon } & \text { Fort McNair } \\ \text { Fort Monmouth } & \text { Fort Stewart } & \text { Pentagon Boiler Plan } \\ \text { Fort Myer } & \text { Fort Wainwright } & \text { Picatinny Arsenal } \\ \text { Fort Riley } & \text { Fort Lee } & \text { Redstone Arsenal } \\ \text { Fort Rucker } & \text { Fort Gillem } & \text { West Point } \\ \text { Fort Sill } & \text { NASA } & \end{array}$

TMS contacted Mr. Henry Studebaker ${ }^{*}$ for information on Navy facilities operating low-NOx burners. Mr. Studebaker provided a list of $12 \mathrm{Navy}$ and Marine installations that had installed LNBs, and appropriate contacts at these locations:

Subase New London
Naval District Washington DC
Naval Air Station Whidbey Island
Naval Station Everett
Naval Subase King's Bay
Naval Shipyard Puget Sound

Subase New London Naval Education and Training Center Marine Corps Air Ground Combat Center Naval Medical Center San Diego Marine Corps Air Station New River Subase Point Loma Naval Amphibious Base San Diego

Mr. Studebaker also provides updated contact information for Mr. Gerry Doddington, ${ }^{\dagger}$ who was the reference listed by CERL for information on U.S. Air

\footnotetext{
* A. Henry Studebaker, P.E. Mechanical Engineer, Naval Facilities Engineering Service Center, $110023^{\text {rd }}$ Ave., Port Hueneme, CA, (805) 982-3524.

† Gerry Doddington, Air Force Civil Engineer Support Agency, 139 Barnes Drive, Suite 1, Tyndall AFB, FL, (850) 283-6343.
} 
Force facilities using LNB technology. Mr. Doddington provided TMS with a list of six Air F orce Bases (AFBs) to contact:

$\begin{array}{ll}\text { Andrews AFB } & \text { McGuire AFB } \\ \text { Brooks AFB } & \text { Robins AFB } \\ \text { Langley AFB } & \text { Vandenburg AFB }\end{array}$

The 32 facilities identified by the CERL combined with the 12 Navy and 6 Air Force facilities combined to provide TMS with a list of 50 facilities to contact, the maximum number of facilities specified in the Statement of Work (SOW). Appendix A lists complete contact information. 


\section{Survey Questionnaire and Data Base Development}

\section{Questionnaire Development}

TMS personnel then created a questionnaire for distribution to the facilities that would elicit responses containing comprehensive information on the boilers and LNBs. The questionnaire was designed to be specific and thorough, but brief, to prevent any undue burden on DOD facility personnel. The survey asked the same seven basic questions suggested in the Research Plan. Appendix B includes the text of the questionnaire.

A data base was also developed by TMS personnel for storing contact information and data contained in the responses to the questionnaire. The data base was designed using Microsoft Access. Its design was similar to the questionnaire format to simplify the data entry process. Appendix $C$ lists the survey results.

\section{Questionnaire Testing and Distribution}

Contact information for each of the 50 facilities was established by telephone contact with the Office of the Director of Public Works (DPW) or equivalent at each facility. The e-mail address and phone number of the DPW or responsible party was recorded in the data base. The DPW or equivalent offices were advised of the pending questionnaire and encouraged to return a completed questionnaire in a timely manner. When redirected by the DPW to a different office, TMS personnel recorded contact information for the recommended office.

Before being distributed to all 50 facilities, the questionnaire was tested on five facilities: Langley AFB, Aberdeen Proving Ground, Fort Belvoir, Fort Benning, and F ort Bragg. The survey was sent to the DPW or equivalent office via e-mail on 5 November 1998. TMS personnel also contacted DPW offices at the facilities by telephone to advise them of the objective and importance of the survey and to verify the e-mail address. The purpose of the test was to identify any changes to the questionnaire or data base that might result in better or more complete reporting of data relevant to the objective of the survey. Unfortunately, TMS 
received only one response - a note from Fort Belvoir indicating that the facility did not, in fact, operate any LNBs. After 2 weeks without further response, it was decided to proceed with the survey without altering the questionnaire. The questionnaire was then emailed to the contact established at each of the other 45 facilities over the period of 24 November to 5 December 1998.

Data from responses to the questionnaire was entered into the data base on receipt by TMS personnel. Some of the responses specified two or more different types of boilers operating LNBs. In such a case, separate records in the data base were used for each type of boiler. If different types of boilers were not specified, but multiple boilers were indicated, only one record was made. If the facility response contained separate answers to the questionnaire for each boiler, multiple records were used. 


\section{Results}

Appendix $C$ lists the survey results. The following sections report the level of response to the questionnaire, a scientific analysis of the data collected, and a subjective analysis of the survey.

\section{Level of Response}

The overall level of response to the survey was low. Twenty-eight facilities responded to the survey:

$\begin{array}{ll}\text { Andrews AFB } & \text { Fort Wainwright } \\ \text { Brooks AFB } & \text { Langley AFB } \\ \text { Fort Belvoir } & \text { Marine Corps Air Ground Combat Center } \\ \text { Fort Benning } & \text { McGuire AFB } \\ \text { Fort Campbell } & \text { Naval Amphibious Base San Diego } \\ \text { Fort Dix } & \text { Naval District Washington DC } \\ \text { Fort Gillem } & \text { Naval Education and Training Center } \\ \text { Fort Gordon } & \text { Naval Medical Center San Diego } \\ \text { Fort Lee } & \text { Naval Shipyard Puget Sound } \\ \text { Fort Lewis } & \text { Naval Station Everett } \\ \text { Fort Monmouth } & \text { Picatinny Arsenal } \\ \text { Fort Riley } & \text { Subase Point Loma } \\ \text { Fort Rucker } & \text { Vandenburg AFB } \\ \text { Fort Stewart } & \text { West Point }\end{array}$

In addition to the facilities not responding, many of the contacts replied with a negative response, i.e., the facility did not operate any boilers with LNBs. The 15 facilities reporting no LNBs are tabulated below, along with notes about the boilers at the facility. 
Table 1. Facilities reporting no LNBs.

\begin{tabular}{|l|l|}
\hline DOD Facility & Notes \\
\hline Brooks AFB & Natural gas boilers \\
\hline Everett, WA & Natural gas boilers \\
\hline Fort Belvoir & Now converting to natural gas operation \\
\hline Fort Benning & Many small boilers \\
\hline Fort Campbell & LNB approved but never received or installed \\
\hline Fort Gillem & Natural gas boilers \\
\hline Fort Lewis & Natural gas boilers \\
\hline Fort Monmouth & Natural gas boilers \\
\hline Fort Rucker & \\
\hline Fort Stewart & \\
\hline Fort Wainwright & Coal-fired boilers \\
\hline Fort Lee & Natural gas boilers \\
\hline Langley AFB & \\
\hline McGuire AFB & Just began retrofit; will complete 12/99 \\
\hline Picatinny Arsenal & LNB installation to begin in April, will complete 12/99 \\
\hline
\end{tabular}

The contact at one of these facilities (Fort Rucker) replied that, even after researching literature on the boilers and querying boiler operations personnel, he was not sure whether his facility had LNBs. He requested that TMS personnel supply him with information to identify which of Fort Rucker's boilers might be equipped with the technology. For the purposes of this survey, Fort Rucker was classified as not having LNBs installed.

Of these 15 facilities, several completed the questionnaire, citing the installation of natural gas boilers as a NOx-reducing technology. For this reason, facilities that completed the questionnaire (but reported no LNBs) were included in the data base.

The remaining 13 facilities supplied responses to the questionnaire indicating the presence of LNBS on facility boilers. The thoroughness of these responses varied, from some that provided detailed answers to each question to some that responded to the questionnaire by returning an existing parallel document such as emissions reports or internal reviews. TMS personnel culled as much relevant data from these documents as possible, but there are gaps in the data for several facilities. The 13 facilities that responded to the questionnaire and reported operating LNBs were: 
Andrews AFB

Fort Dix

Fort Gordon

Fort Riley

Marine Corps Air Ground Combat Center

Naval Amphibious Base

Naval District Washington DC
Naval Education and Training Center

Naval Medical Center

Naval Shipyard Puget Sound

Subase Point Loma

Vandenburg AFB

West Point Military Academy

\section{Summary of Findings}

The 13 facilities returning a positive response to the questionnaire provided data to account for 16 records in the data base. Facilities responding with completed questionnaires but operating natural gas burners not equipped with nominal LNBs provided data for another 10 records.

The resulting 26-record data base does not conclusively indicate a problem with LNB operations in the field, but does indicate some concern on the part of boiler operating personnel. Ten of the 16 LNB records, or 62.5 percent, indicate that problems have been encountered during installation, commissioning, or operation. The low level of response indicates that facilities may have responded only if they had complaints about the LNBs. Even if all 22 nonresponding facilities have LNBs operating without problems, at least 26 percent of the possible LNB facilities have encountered significant problems. The type of problem most frequently mentioned in the 16 LNB records is operational difficulties, followed by commissioning and installation problems.

The most frequently cited operational problem seems to be compromised emissions performance. The LNBs do not seem to be delivering the reduced NOx levels promised. In addition, several facilities report problems with flameout or other flame problems. At least eight of the 16 LNB facilities reported some type of flame control problems relating to either $\mathrm{O}_{2}$ trim, gas line pressure, control system, or flame shape, with $\mathrm{O}_{2}$ trim being the most frequently blamed cause. Other operational problems mentioned include electrode failure, excessively high superheat temperatures, Flue Gas Recirculator (FGR) problems, and excessive vibration during operation. The facilities indicated that some problems were solved by modifying the equipment in the field, but the result is a customized burner that is not easy to replace. 
Table 2. Operational problems and possible solutions.

\begin{tabular}{|l|l|}
\hline Problem & Possible Solution \\
\hline $\begin{array}{l}\text { NOx levels not reduced to correct } \\
\text { levels }\end{array}$ & $\begin{array}{l}\text { Install inlet damper to throttle air opening and increase FGR flow } \\
\text { Change burner tips for different flame shape }\end{array}$ \\
\hline Flameout & $\begin{array}{l}\text { Resize orifice in pressure regulator to stabilize gas pressure } \\
\text { Adjust location or flow of FGR } \\
\text { Add screen around register }\end{array}$ \\
\hline Premature electrode failure & Cyclical loading problems - may need to downsize burner \\
\hline Excessive Vibration & Install plates in boiler breaching to reduce vortices \\
\hline $\mathrm{O}_{2}$ control & Add $\mathrm{O}_{2}$ trim system \\
\hline
\end{tabular}

Installation and commissioning problems with the burners seem to be related to each other. Three of the 16 NOx facilities complained about the failure of installation contractors to help throughout the troubleshooting process. However, one response actually commended the installation contractor for quality work and perseverance. Two facilities also mentioned that problems could be traced to the fact that, with improved efficiency, the boilers became oversized for the application. Other installation and commissioning problems cited included problems with two boilers sharing a common stack, improper burner tips, and problems interfacing new equipment with dirty equipment.

Table 3. Installation and commissioning problems and possible solutions.

\begin{tabular}{|l|l|}
\hline Problem & Possible Solution \\
\hline Ignition problems in common stack configuration & Install stack dampers \\
\hline Insufficient support by installation contractor & $\begin{array}{l}\text { Establish higher standards for contractors } \\
\text { Define contractual requirements for support through } \\
\text { commissioning stages } \\
\text { Use contractors with specific experience with LNB } \\
\text { installation }\end{array}$ \\
\hline $\begin{array}{c}\text { Field modification of burners makes replacement } \\
\text { difficult }\end{array}$ & $\begin{array}{c}\text { Establish communication with manufacturer to voice } \\
\text { concerns }\end{array}$ \\
\hline
\end{tabular}

In addition to the data entered into the data base, the survey provided an opportunity for informal discussion with site staff on LNB successes and shortcomings. In the course of conducting the survey, TMS personnel had the opportunity to interact via telephone and e-mail with boiler operations personnel at almost all of the 50 facilities contacted in the survey. It is evident that some facilities with valuable input on LNB operation failed to return a questionnaire or were excessively brief in responding to the questionnaire. Some subjective observations made by TMS personnel based on conversations with DOD facility personnel were: 
1. With increasingly stringent emissions limits, low NOx technology is becoming a preferred option when boiler modernization projects are undertaken at DOD facilities.

2. Many facilities are also retrofitting boilers to operate on natural gas instead of fuel oil or coal, for the same reasons.

3. Retrofitting boilers with LNB technology has resulted in much more frequent operational problems than installing new boilers designed to operate with LNBs.

4. Flameout is a common problem in boilers that have been retrofitted with LNBS. Opinions on exactly why the flameout occurs seem to be as numerous as the facilities.

5. There is a widespread lack of knowledge in the field about LNB technology, and what exactly constitutes a "Low-NOx Burner."

6. Contractors are frequently blamed for problems with boiler operations after installation of LNBs.

7. Problems with Flue Gas Recirculation (FGR) are frequent after installation of LNB equipment. FGR design or flow rate typically need to be adjusted to achieve reduced NOx emissions. 


\section{Conclusions and Recommendations}

The data collected in this survey did not conclusively show that LNBs are chronically problematic, but a majority of the LNB-equipped boilers recorded in the data base reported problems with operation, installation, or commissioning. The most frequently reported problems were the failure of the unit to reduce NOx emissions to the level promised, and flame instability caused by a variety of factors. Although survey respondents reported numerous innovative attempts to solve these problems, the LNBs seem to be a source of significant frustration for boiler operations personnel.

To further pinpoint and minimize problems with the technology, it is recommended that future data collection begin by choosing several LNB facilities to participate in a dialogue in which problems and solutions can be discussed in greater detail. It may be useful to examine similarities and differences in the operating environments of different facilities, the configuration of multiple-boiler systems, and the exact installation techniques best suited to LNB retrofits.

The low level of response indicates that a written or electronic survey may not be the most effective method of collecting LNB operation information from the field. Facility personnel are reluctant to devote significant time or effort to written questions. Also, a general survey limits the ability to focus on specific problems at specific sites. A more effective and efficient method of collecting information on LNB operation may be to focus on approximately five facilities and develop a detailed history and analysis of LNB operation at each site. Problems could be investigated thoroughly, and a protocol for solving recurring LNB problems could be developed. 


\title{
Appendix A: Contacts
}

\author{
U.S. Military Academy West Point (U.S. Army) \\ Fort Jackson (U.S. Army) \\ USMA West Point \\ Director of Public Works \\ West Point, NY 10996-1592 \\ ATTN: ATZJ-PW \\ Environmental Group \\ Fort Jackson, SC 29207-5650 \\ Andrews AFB (U.S. Air Force) \\ Fort Drum (U.S. Army) \\ Mechanical Engineer \\ Director of Public Works \\ 89 CES/CEOE \\ B3446 Tennessee Ave. \\ Andrews AFB, MD 20762-4803 \\ 85 First Street West \\ ATTN: AFZS-PW \\ Fort Drum, NY 13602-5097 \\ Fort Lee (U.S. Army) \\ Fort Hood (U.S. Army) \\ Director of Public Works \\ Director of Public Works \\ ATTN: ATZM-E \\ ATTN: AFZF-PW \\ 1816 Shop Road \\ Fort Hood, TX 76544-5057 \\ Fort Lee, VA 23801-1604 \\ Redstone Arsenal (U.S. Army) \\ ATTN: AMSMI-RA-DPW \\ Redstone Arsenal, AL 35898-5340 \\ Director of Public Works \\ Fort Lewis (U.S. Army) \\ Director of Public Works \\ ATTN: AFZH-PW, MS17 \\ Box 339500 \\ Fort Lewis, WA 98433-9500 \\ Fort Benning (U.S. Army) \\ Director of Public Works \\ USAIC, DPW, Meloy Hall, Bldg. 6 \\ Fort Benning, GA 31905 \\ Fort Gordon (U.S. Army) \\ Director of Public Works \\ ATTN: ATZH-DI \\ Fort Gordon, GA 30905-5040 \\ Naval Station Everett (U.S. Navy) \\ Energy Manager \\ Naval Station Everett \\ Everett, WA \\ Aberdeen Proving Ground (U.S. Army) \\ Director of Public Works \\ ATTN: STEAP-FE \\ Aberdeen Proving Ground, MD 21005-5055 \\ Naval Medical Center San Diego (U.S. Navy) \\ General Foreman \\ Fort Carson (U.S. Army) \\ Naval Medical Center \\ Director of Public Works \\ San Diego, CA \\ ATTN: AFZC-DPW \\ 805 Tevis Street \\ Point Loma (U.S. Navy) \\ Fort Carson, CO 80913-4001 \\ General Foremen \\ San Diego, CA \\ Fort Rucker (U.S. Army) \\ Naval Amphloious Base (U.S. Navy) \\ Director of Public Works \\ General Formean \\ ATTN: ATZQ-DPW \\ San Diego, CA
}


Fort McNair (U.S. Army)

U.S. Army Military District of Washington

Chief, Environmental Support Division

ATTN: ANEN-ES

Fort McNair

Washington, DC 20319-5050

Fort Riley (U.S. Army)

Director of Public Works

ATTN: AFZN-PW

Fort Riley, KS 66442-6000

Fort Belvoir (U.S. Army)

Director of Public Works

ATTN: ANFB-PW

Fort Belvoir, VA 22060-5130

Fort George G. Meade (U.S. Army)

Director of Public Works

ATTN: ANME-PW

Fort George G. Meade, MD 20755-5115

Fort Gillem (U.S. Army)

Forest Park, GA

Fort Knox (U.S. Army)

Director of Public Works

U.S. Army Armor Center and Fort Knox

ATTN: ATZK-PW

Fort Knox, KY 40121-5000

Picatinny Arsenal (U.S. Army)

Director of Public Works

ATTN: AMSTA-AR-PW, BIdg. 3002

Picatinny Arsenal, NJ 07806-5000

Naval Air Station Whidbey Island (U.S. Navy)

Utility Director

Naval Air Station Whidbey Island

Whidbey Island, WA

Pentagon Boiler Plant (U.S. Army)

Naval Shipyard Puget Sound (U.S. Navy)

Mechanical Engineer

Puget Sound, WA
Brooks AFB (U.S. Air Force)

Superintendent of HVAC

Brooks AFB

San Antonio, TX

Fort Campbell (U.S. Army)

Acting Director of Public Works

ATTN: AFZB-DPW

16th \& Ohio Street

Fort Campbell, KY 42223-5130

Vandenburg AFB (U.S. Air Force)

Mechanical Engineer

Vandenburg AFB

Lompoc, CA

Langley AFB (U.S. Air Force)

Fort Monmouth (U.S. Army)

Director of Public Works

USA Garrison

ATTN: SELFM-PW

Fort Monmouth, NJ 07703-5108

Fort Leonard Wood (U.S. Army)

Director of Public Works

ATTN: ATZT-DPW

Fort Leonard Wood, MO 65473-5000

Subase New London (U.S. Navy)

Utility Director

New London, CT

Fort Stewart (U.S. Army)

Director of Public Works

ATTN: AFZP-DE

Bldg. 1101, Utility Street

Fort Stewart, GA 31314-5000

Robins AFB (U.S. Air Force)

Fort Eustis (U.S. Army)

Director of Public Works

USATCFE

Washington Blvd., Bldg. 1407

Fort Eustis, VA 23604-5306 
Fort Myer (U.S. Army)

Director of Public Works

ATTN: ANMY-PWZ

Fort Myer, VA 22211-5050

Fort Bragg (U.S. Army)

Director of Public Works

ATTN: AFZA-PW

Fort Bragg, NC 28307-5000

MCAS New River (DOD)

Assistant Facility Manager

New River, NC

Naval Education and Training Center (U.S. Navy)

Public Works Engineering Division

Supervisor Planning

Naval Education and Training Center , RI

Carlisle Barracks (U.S. Army)

Director of Public Works

ATTN: ATZE-DPW

330 Engineer Avenue

Carlisle Barracks, PA 17013-5002

Fort Dix (U.S. Army)

Director of Public Works

ATTN: AFRC-FA-PW, Bldg. 5320

Fort Dix, NJ 08640-5500

Naval Subase Kings Bay (U.S. Navy)

Energy Engineer

Kings Bay, GA

Naval District Washington DC (U.S. Navy)

Mechanical Engineer

Washington, DC

Fort Sill (U.S. Army)

Director of Public Works

ATTN: ATZR-E

Fort Sill, OK 73503-5100

Fort Wainwright (U.S. Army)

Director of Public Works

ATTN: APVR-WPW

Fort Wainwright, AK 99703-6500 


\section{Appendix B: Questionnaire}

The U.S. Army Construction Engineering Research Laboratory (CERL) has commissioned a survey of Department of Defense facilities that have installed burners to control emissions of nitrogen oxides (NOx) on gas- and oil-fired boilers. Your facility has been identified as operating a boiler (or boilers) that use this important technology.

Below is a questionnaire requesting information on the performance of these boilers. Please take a few minutes to answer each inquiry completely. Insert answers to the questions bel ow and feel free to add further comments or information that you think is important under Question 7 at the end of the questionnaire. Please respond by Friday, J anuary 15, 1999.

If you have questions or problems, call me at (301) 670-6390, Ext. 34. Thank you for your help.

Peter Herz

Technology and Management Services, Inc.

18757 North Frederick Road

Gaithersburg, MD 20879

(301) 670-1942 (FAX)

pherz@tms-hq.com 


\section{CERL Low NOx Burner Survey}

Question 1. Please identify the characteristics of the boiler(s):

Boiler Type:

Boiler Size MWe:

and/or

Boiler Size MBtu/hr of Steam

Boiler Fuel:

Boiler Description:

Boiler Vendor:

Serial Number:

Date Installed:

Number of Burners:

Burner Operating System:

Other Burner Operating System:

Question 2. Please state your air permit requirements, including NOx emission limit.

Requirements:

NOx Limit: $\quad$ Ib/mBtu 
Question 3. Please identify any recent major modifications, including low NOx burners (LNB).

LNB Type:

LNB Vendor:

LNB Installation Date:

Date of Major Modification:

Other Modifications:

Flame Scanner (Yes or No):

Flame Scanner Vendor:

Question 4. Please describe the LNB installation problems you have experienced, the solutions that you have implemented to solve the problems, and whether these solutions have been successful.

Installation Problems:

Installation Solutions: 
Question 5. Please describe the LNB commissioning problems you have experienced, the solutions that you have implemented to solve the problems, and whether these solutions have been successful.

Commissioning Problems:

Commissioning Solutions:

Question 6. Please describe the LNB operational problems you have experienced, the solutions that you have implemented to solve the problems, and whether these solutions have been successful.

Operational Problems:

Operational Solutions: 
Question 7. Are there any other issues or problems regarding boiler safety that you can identify? 


\section{Appendix C: Survey Results}

\begin{tabular}{|c|c|c|c|}
\hline Facility ID & 27 & Boiler Descriptions & Copper Fin II \\
\hline Facility Name & Fort Benning & Boiler Serial No. & \\
\hline Boiler Type & Multiple Boilers (over 50) & Date Boiler Installed & \\
\hline Other Type & & LNB Install Date & \\
\hline Boiler Size & 300 to $1800 \mathrm{Mbtu} / \mathrm{hr}$ & LNB Vendor & \\
\hline Boiler Fuel & Natural Gas & Date of Major Mod & \\
\hline Combination Fuels & & LNB Type & \\
\hline Burner System & & NOx Emission Limit & 0 \\
\hline Other Burner System & & Flame Scanner? & No \\
\hline No. of Burners & & $\begin{array}{l}\text { Flame Scanner Ven- } \\
\text { dor }\end{array}$ & \\
\hline Boiler Vendor & Lochinvar & $\begin{array}{l}\text { Permit Require- } \\
\text { ments }\end{array}$ & $\begin{array}{l}\text { The state of Georgia does not have a limit on } \\
\text { NOx emissions for boilers of these sizes }\end{array}$ \\
\hline \multicolumn{4}{|c|}{ Description of other Modifications } \\
\hline \multicolumn{4}{|c|}{ Description of Commissioning Problems } \\
\hline \multicolumn{4}{|c|}{ Description of Commissioning Solutions } \\
\hline \multicolumn{4}{|c|}{ Description of Installation Problems } \\
\hline \multicolumn{4}{|c|}{ The manufacturer provided an extraordinary class on the O\&M of the boilers at the conclusion of the project. } \\
\hline \multicolumn{4}{|c|}{ Description of Installation Solutions } \\
\hline \multicolumn{4}{|c|}{$\begin{array}{l}\text { The instructor disassembled and reassembled the boilers in the on site instruction. There are not any other issues or problems on these } \\
\text { boilers that we have any comments on. }\end{array}$} \\
\hline \multicolumn{4}{|c|}{ Description of Operational Problems } \\
\hline \multicolumn{4}{|c|}{ Description of Operational Solutions } \\
\hline \multicolumn{4}{|l|}{ Other Comments } \\
\hline $\begin{array}{l}\text { Fort Benning has recently } \mathrm{c} \\
\text { buildings. All } 80 \text { or so boile }\end{array}$ & $\begin{array}{l}\text { npleted a project in which w } \\
\text { were Lochinvar Copper Fin }\end{array}$ & $\begin{array}{l}\text { shut down a central plant and } \\
\text { type which are low NOx }\left({ }^{*} 9.9\right.\end{array}$ & $\begin{array}{l}\text { stalled about } 80 \text { new boilers in about } 40 \\
\text { m, I think). }\end{array}$ \\
\hline
\end{tabular}

\begin{tabular}{|c|c|c|c|c|c|c|c|}
\hline Rank & \multicolumn{2}{|c|}{ First Name } & Last Name & & \multicolumn{3}{|l|}{ Title } \\
\hline & & & & & \multicolumn{3}{|c|}{ USAIC, Director of Public Works, Melody Hall } \\
\hline \multicolumn{4}{|c|}{ Address } & \multicolumn{2}{|l|}{ City } & State & Postal Code \\
\hline \multicolumn{4}{|c|}{ USAIC, DPW, Meloy Hall, BIdg. 6} & \multicolumn{2}{|l|}{ Fort Benning } & GA & 31905 \\
\hline \multicolumn{2}{|c|}{ Work Phone } & Ext. & Fax Number & \multicolumn{4}{|c|}{ E-Mail Address } \\
\hline
\end{tabular}




\begin{tabular}{|c|c|c|c|}
\hline Facility ID & 39 & Boiler Descriptions & $\begin{array}{l}\text { Field Erected, side burner installed, original coal } \\
\text { fired }\end{array}$ \\
\hline Facility Name & Fort Dix & Boiler Serial No. & \\
\hline Boiler Type & Water Tube & Date Boiler Installed & \\
\hline Other Type & & LNB Install Date & $01 / 22 / 98$ \\
\hline Boiler Size & $50,000 \mathrm{lbs} / \mathrm{hr}$ & LNB Vendor & S.T. Johnson \\
\hline Boiler Fuel & Natural Gas & Date of Major Mod & \\
\hline Combination Fuels & & LNB Type & $\begin{array}{l}\text { Forced Draft, Gas Only, Recirculating exhaust } \\
\text { gas }\end{array}$ \\
\hline Burner System & & NOx Emission Limit & $0.1 \mathrm{lb} / \mathrm{Mbtu}$ \\
\hline Other Burner System & & Flame Scanner? & yes \\
\hline No. of Burners & 2 per boiler & Flame Scanner Vendor & Fireye \#45UV5 \\
\hline Boiler Vendor & Keeler & Permit Requirements & \\
\hline \multicolumn{4}{|c|}{ Description of other Modifications } \\
\hline \multicolumn{4}{|c|}{ Description of Commissioning Problems } \\
\hline \multicolumn{4}{|c|}{ Description of Commissioning Solutions } \\
\hline \multicolumn{4}{|c|}{ Description of Installation Problems } \\
\hline \multicolumn{4}{|l|}{ None } \\
\hline \multicolumn{4}{|c|}{ Description of Installation Solutions } \\
\hline \multicolumn{4}{|c|}{ Description of Operational Problems } \\
\hline \multicolumn{4}{|c|}{ Description of Operational Solutions } \\
\hline Other Comments & & & \\
\hline
\end{tabular}

\begin{tabular}{|c|c|c|c|c|c|c|c|}
\hline Rank & \multicolumn{2}{|c|}{ First Name } & Last Name & & \multicolumn{3}{|l|}{ Title } \\
\hline & & & & & \multicolumn{3}{|c|}{ Director of Public Works } \\
\hline \multicolumn{4}{|c|}{ Address } & \multicolumn{2}{|l|}{ City } & State & Postal Code \\
\hline \multicolumn{4}{|c|}{ ATTN: AFRC-FA-PW, Bldg. 5320} & \multicolumn{2}{|l|}{ Fort Dix } & NJ & 08640-5500 \\
\hline \multicolumn{2}{|c|}{ Work Phone } & Ext. & Fax Number & \multicolumn{4}{|c|}{ E-Mail Address } \\
\hline
\end{tabular}




\begin{tabular}{|c|c|c|c|}
\hline Facility ID & 2 & Boiler Descriptions & Low-pressure Scotch Marine \\
\hline Facility Name & Gillem & Boiler Serial No. & 01096778 \& 01096777 \\
\hline Boiler Type & Other (2 Boilers) & Date Boiler Installed & $1 / 1 / 77$ \\
\hline Other Type & & LNB Install Date & \\
\hline Boiler Size & $10,205,800 \mathrm{Mbtu} / \mathrm{hr}$ & LNB Vendor & \\
\hline Boiler Fuel & Natural Gas & Date of Major Mod & \\
\hline Combination Fuels & Propane & LNB Type & None \\
\hline Burner System & Other & NOx Emission Limit & \\
\hline Other Burner System & Fire Eye EB 700 & Flame Scanner? & No \\
\hline No. of Burners & 1 & Flame Scanner Vendor & \\
\hline Boiler Vendor & Cleaver Brooks & Permit Requirements & 49.9 tpy (all sources) for Nox \\
\hline \multicolumn{4}{|c|}{ Description of other Modifications } \\
\hline \multicolumn{4}{|c|}{ Description of Commissioning Problems } \\
\hline \multicolumn{4}{|l|}{$\mathrm{N} / \mathrm{A}$} \\
\hline \multicolumn{4}{|c|}{ Description of Commissioning Solutions } \\
\hline \multicolumn{4}{|c|}{ Description of Installation Problems } \\
\hline \multicolumn{4}{|l|}{$\mathrm{N} / \mathrm{A}$} \\
\hline \multicolumn{4}{|c|}{ Description of Installation Solutions } \\
\hline \multicolumn{4}{|c|}{ Description of Operational Problems } \\
\hline \multicolumn{4}{|l|}{$\mathrm{N} / \mathrm{A}$} \\
\hline \multicolumn{4}{|c|}{ Description of Operational Solutions } \\
\hline \multicolumn{4}{|l|}{ Other Comments } \\
\hline None & & & \\
\hline
\end{tabular}

\begin{tabular}{|l|l|l|l|l|l|}
\hline Rank & First Name & Last Name & \multicolumn{3}{l|}{ Title } \\
\hline & \multicolumn{5}{|l|}{} \\
\hline Address & & City & State & Postal Code \\
\hline \multicolumn{7}{|l|}{} & Forest Park & GA & \\
\hline Work Phone & Ext. & Fax Number & E-Mail Address & & \\
\hline & & & & \\
\hline
\end{tabular}




\begin{tabular}{|c|c|c|c|}
\hline Facility ID & 24 & Boiler Descriptions & \\
\hline Facility Name & Fort Gordon & Boiler Serial No. & \\
\hline Boiler Type & $\begin{array}{l}10 \text { Natural Gas } \\
\text { boilers, no LNB's }\end{array}$ & Date Boiler Installed & $1965-97$ \\
\hline Other Type & & LNB Install Date & \\
\hline Boiler Size & $13,800-35,850 \mathrm{lbs} / \mathrm{hr}$ & LNB Vendor & \\
\hline Boiler Fuel & Natural Gas & Date of Major Mod & \\
\hline Combination Fuels & \#2 fuel oil & LNB Type & \\
\hline Burner System & & NOx Emission Limit & 0 \\
\hline Other Burner System & & Flame Scanner? & No \\
\hline No. of Burners & & Flame Scanner Vendor & \\
\hline Boiler Vendor & $\begin{array}{l}\text { Cleaver Brooks, } \\
\text { Nebraska Boiler, } \\
\text { Erie City Ironwor }\end{array}$ & Permit Requirements & $\begin{array}{l}\text { The emission limits for NOx in the new boilers is: } \\
0.20 \text { lbs per MBtu gaseous fuel; } 0.30 \mathrm{lbs} \text { per } \\
\text { MBtu liquid fossil fuel. Older boilers: } 100 \text { tons } \\
\text { per year or not to exceed } 100 \text { micrograms per } \\
\text { cubic meter at ground level. }\end{array}$ \\
\hline \multicolumn{4}{|c|}{ Description of other Modifications } \\
\hline \multicolumn{4}{|c|}{ Description of Commissioning Problems } \\
\hline \multicolumn{4}{|l|}{$N / A$} \\
\hline \multicolumn{4}{|c|}{ Description of Commissioning Solutions } \\
\hline \multicolumn{4}{|c|}{ Description of Installation Problems } \\
\hline \multicolumn{4}{|l|}{$N / A$} \\
\hline \multicolumn{4}{|c|}{ Description of Installation Solutions } \\
\hline \multicolumn{4}{|c|}{ Description of Operational Problems } \\
\hline \multicolumn{4}{|l|}{$\mathrm{N} / \mathrm{A}$} \\
\hline \multicolumn{4}{|c|}{ Description of Operational Solutions } \\
\hline \multicolumn{4}{|l|}{ Other Comments } \\
\hline$N / A$ & & & \\
\hline
\end{tabular}

\begin{tabular}{|l|l|l|l|l|l|}
\hline Rank & First Name & \multicolumn{2}{l|}{ Last Name } & \multicolumn{2}{l|}{ Title } \\
\hline \multicolumn{7}{|l|}{} & \multicolumn{3}{l|}{ Director of Public Works } \\
\hline Address & & City & State & Postal Code \\
\hline ATTN: ATZH-DI & Fort Gordon & GA & $30905-5040$ \\
\hline Work Phone & Ext. & Fax Number & E-Mail Address & \\
\hline & & & & \\
\hline
\end{tabular}




\begin{tabular}{|c|c|c|c|}
\hline Facility ID & 14 & Boiler Descriptions & Scotch Marine Firetube \\
\hline Facility Name & Fort Lewis & Boiler Serial No. & 1-L-82519, 2-L-82518, 3-L-82517 \\
\hline Boiler Type & Five Boilers & Date Boiler Installed & $7 / 21 / 87$ \\
\hline Other Type & & LNB Install Date & \\
\hline Boiler Size & $\begin{array}{l}2 \times 20.92,2 \times 13.40 \\
1 \times 25.11 \mathrm{MBtu} / \mathrm{hr}\end{array}$ & LNB Vendor & \\
\hline Boiler Fuel & Natural Gas & Date of Major Mod & \\
\hline Combination Fuels & $\begin{array}{l}\text { and \#2 } 0.01 \% \text { sulfur) } \\
\text { Fuel Oil }\end{array}$ & LNB Type & None \\
\hline Burner System & Honeywell & NOx Emission Limit & 0 \\
\hline Other Burner System & CBs & Flame Scanner? & No \\
\hline No. of Burners & 1 each & Flame Scanner Vendor & \\
\hline Boiler Vendor & Clever Brooks & Permit Requirements & $\begin{array}{l}0.1 \mathrm{lbs} / \mathrm{MBtu} \text { for Natural gas and } 0.3 \mathrm{lbs} / \mathrm{MBtu} \text { on } \\
\text { fuel oil }\end{array}$ \\
\hline \multicolumn{4}{|c|}{ Description of other Modifications } \\
\hline \multicolumn{4}{|c|}{ Description of Commissioning Problems } \\
\hline \multicolumn{4}{|l|}{ None } \\
\hline \multicolumn{4}{|c|}{ Description of Commissioning Solutions } \\
\hline \multicolumn{4}{|l|}{$\mathrm{N} / \mathrm{A}$} \\
\hline \multicolumn{4}{|c|}{ Description of Installation Problems } \\
\hline \multicolumn{4}{|l|}{ None } \\
\hline \multicolumn{4}{|c|}{ Description of Installation Solutions } \\
\hline \multicolumn{4}{|l|}{$\mathrm{N} / \mathrm{A}$} \\
\hline \multicolumn{4}{|c|}{ Description of Operational Problems } \\
\hline \multicolumn{4}{|l|}{ None } \\
\hline \multicolumn{4}{|c|}{ Description of Operational Solutions } \\
\hline \multicolumn{4}{|l|}{$\mathrm{N} / \mathrm{A}$} \\
\hline \multicolumn{4}{|l|}{ Other Comments } \\
\hline None & & & \\
\hline
\end{tabular}

\begin{tabular}{|c|c|c|c|c|c|c|c|}
\hline Rank & \multicolumn{2}{|c|}{ First Name } & Last Name & & \multicolumn{3}{|l|}{ Title } \\
\hline & & & & & \multicolumn{3}{|c|}{ Director of Public Works } \\
\hline \multicolumn{4}{|c|}{ Address } & \multicolumn{2}{|l|}{ City } & State & Postal Code \\
\hline \multicolumn{4}{|c|}{$\begin{array}{l}\text { ATTN: AFZH-PW, MS17 } \\
\text { Box } 339500\end{array}$} & \multicolumn{2}{|l|}{ Fort Lewis } & WA & $98433-9500$ \\
\hline \multicolumn{2}{|c|}{ Work Phone } & Ext. & Fax Number & \multicolumn{4}{|c|}{ E-Mail Address } \\
\hline
\end{tabular}




\begin{tabular}{|c|c|c|c|}
\hline Facility ID & 3 & Boiler Descriptions & \\
\hline Facility Name & $\begin{array}{l}\text { Fort George G. } \\
\text { Meade }\end{array}$ & Boiler Serial No. & \\
\hline Boiler Type & Six Boilers & Date Boiler Installed & \\
\hline Other Type & & LNB Install Date & \\
\hline Boiler Size & $\begin{array}{l}(2 \times 48,000) \\
(3 \times 13,800),(9,000) \\
\text { Mbtu/hr }\end{array}$ & LNB Vendor & N/A \\
\hline Boiler Fuel & No. 2 Oil & Date of Major Mod & \\
\hline Combination Fuels & & LNB Type & $\mathrm{N} / \mathrm{A}$; may install LNBs in future \\
\hline Burner System & & NOx Emission Limit & \\
\hline Other Burner System & & Flame Scanner? & No \\
\hline No. of Burners & & Flame Scanner Vendor & \\
\hline Boiler Vendor & & Permit Requirements & \\
\hline \multicolumn{4}{|c|}{ Description of other Modifications } \\
\hline \multicolumn{4}{|l|}{$\mathrm{N} / \mathrm{A}$} \\
\hline \multicolumn{4}{|c|}{ Description of Commissioning Problems } \\
\hline \multicolumn{4}{|l|}{$N / A$} \\
\hline \multicolumn{4}{|c|}{ Description of Commissioning Solutions } \\
\hline \multicolumn{4}{|c|}{ Description of Installation Problems } \\
\hline \multicolumn{4}{|l|}{$\mathrm{N} / \mathrm{A}$} \\
\hline \multicolumn{4}{|c|}{ Description of Installation Solutions } \\
\hline \multicolumn{4}{|c|}{ Description of Operational Problems } \\
\hline \multicolumn{4}{|l|}{$\mathrm{N} / \mathrm{A}$} \\
\hline \multicolumn{4}{|c|}{ Description of Operational Solutions } \\
\hline \multicolumn{4}{|l|}{ Other Comments } \\
\hline None & & & \\
\hline
\end{tabular}

\begin{tabular}{|l|l|l|l|l|l|}
\hline Rank & First Name & \multicolumn{2}{l|}{ Last Name } & \multicolumn{2}{l|}{ Title } \\
\hline & \multicolumn{5}{|l|}{ Director of Public Works } \\
\hline Address & & City & State & Postal Code \\
\hline ATTN: ANME-PW & & Fort George G. Meade & MD & $20755-5115$ \\
\hline Work Phone & Ext. & Fax Number & E-Mail Address & \\
\hline & & & &
\end{tabular}




\begin{tabular}{|c|c|c|c|}
\hline Facility ID & 5 & Boiler Descriptions & $\begin{array}{l}\text { Field erected, multiburner, balanced draft with } \\
\text { tubular air heater }\end{array}$ \\
\hline Facility Name & Picatinny Arsenal & Boiler Serial No. & $6899 \& 6901$ \\
\hline Boiler Type & $\begin{array}{l}\text { Water Tube }(2 \\
\text { identical boilers) }\end{array}$ & Date Boiler Installed & $1 / 1 / 54$ \\
\hline Other Type & & LNB Install Date & \\
\hline Boiler Size & & LNB Vendor & burners by January 2000 \\
\hline Boiler Fuel & Natural Gas & Date of Major Mod & \\
\hline Combination Fuels & \#6 Fuel Oil & LNB Type & $\begin{array}{l}\text { Both boilers are schedule to be equipped with } \\
\text { new }\end{array}$ \\
\hline Burner System & $\begin{array}{l}\text { Natural Gas or \#6 } \\
\text { fuel oil }\end{array}$ & NOx Emission Limit & \\
\hline Other Burner System & & Flame Scanner? & No \\
\hline No. of Burners & 4 & Flame Scanner Vendor & \\
\hline Boiler Vendor & $\begin{array}{l}\text { Combustion } \\
\text { Engineering Co. }\end{array}$ & Permit Requirements & $\begin{array}{l}0.100 \mathrm{lb} / \mathrm{Mbtu} \text { for Natural Gas; } 0.280 \mathrm{lb} / \mathrm{Mbtu} \text { for } \\
\text { Residual Fuel Oil }\end{array}$ \\
\hline \multicolumn{4}{|c|}{ Description of other Modifications } \\
\hline \multicolumn{4}{|c|}{ Description of Commissioning Problems } \\
\hline \multicolumn{4}{|l|}{$\mathrm{N} / \mathrm{A}$} \\
\hline \multicolumn{4}{|c|}{ Description of Commissioning Solutions } \\
\hline \multicolumn{4}{|l|}{$\mathrm{N} / \mathrm{A}$} \\
\hline \multicolumn{4}{|c|}{ Description of Installation Problems } \\
\hline \multicolumn{4}{|l|}{$\mathrm{N} / \mathrm{A}$} \\
\hline \multicolumn{4}{|c|}{ Description of Installation Solutions } \\
\hline \multicolumn{4}{|l|}{$\mathrm{N} / \mathrm{A}$} \\
\hline \multicolumn{4}{|c|}{ Description of Operational Problems } \\
\hline \multicolumn{4}{|l|}{$N / A$} \\
\hline \multicolumn{4}{|c|}{ Description of Operational Solutions } \\
\hline \multicolumn{4}{|l|}{$\mathrm{N} / \mathrm{A}$} \\
\hline \multicolumn{4}{|l|}{ Other Comments } \\
\hline $\mathrm{N} / \mathrm{A}$ & & & \\
\hline
\end{tabular}

\begin{tabular}{|c|c|c|c|c|c|c|}
\hline Rank & \multicolumn{2}{|c|}{ First Name } & Last Name & & \multicolumn{2}{|c|}{ Title } \\
\hline & & & \multicolumn{4}{|c|}{ Director of Public Works } \\
\hline \multicolumn{4}{|c|}{ Address } & City & State & Postal Code \\
\hline \multicolumn{4}{|c|}{$\begin{array}{l}\text { Picatinny Arsenal } \\
\text { ATTN: AMSTA-AR-PW, Bldg. } 3002\end{array}$} & Picatinny Arsenal & NJ & $07806-5000$ \\
\hline \multicolumn{2}{|c|}{ Work Phone } & Ext. & Fax Number & \multicolumn{3}{|c|}{ E-Mail Address } \\
\hline
\end{tabular}




\begin{tabular}{|c|c|c|c|}
\hline Facility ID & 10 & Boiler Descriptions & Water Tube Fire Box Constructed (2 each) \\
\hline Facility Name & Fort Riley & Boiler Serial No. & Nat. Board \# 19312; Nat. Board \# 19313 \\
\hline Boiler Type & $\begin{array}{l}\text { Two boilers-Babcock } \\
\& \text { Wilcox type "FF" }\end{array}$ & Date Boiler Installed & 1955 \\
\hline Other Type & & LNB Install Date & $9 / 1 / 98$ \\
\hline Boiler Size & $27.5 \mathrm{Mbtu} / \mathrm{hr}$ each & LNB Vendor & Coen \\
\hline Boiler Fuel & Natural Gas & Date of Major Mod & $9 / 1 / 98$ \\
\hline Combination Fuels & Oil & LNB Type & Forced Draft \\
\hline Burner System & $\begin{array}{l}\text { Honeywell SCAN } \\
3000 \text { Automatic } \\
\text { Combustion Control }\end{array}$ & NOx Emission Limit & .10 \\
\hline Other Burner System & & Flame Scanner? & No \\
\hline No. of Burners & 1 each & Flame Scanner Vendor & Fireye \\
\hline Boiler Vendor & Babcock \& Wilcox & Permit Requirements & No stated limits. $0.10 \mathrm{lbs} / \mathrm{Mbtu}$ for natural gas \\
\hline \multicolumn{4}{|c|}{ Description of other Modifications } \\
\hline \multicolumn{4}{|c|}{ Description of Commissioning Problems } \\
\hline \multicolumn{4}{|l|}{ None } \\
\hline \multicolumn{4}{|c|}{ Description of Commissioning Solutions } \\
\hline \multicolumn{4}{|c|}{ Description of Installation Problems } \\
\hline \multicolumn{4}{|l|}{ None } \\
\hline \multicolumn{4}{|c|}{ Description of Installation Solutions } \\
\hline \multicolumn{4}{|c|}{ Description of Operational Problems } \\
\hline \multicolumn{4}{|l|}{ None } \\
\hline \multicolumn{4}{|c|}{ Description of Operational Solutions } \\
\hline Other Comments & & & \\
\hline
\end{tabular}

\begin{tabular}{|l|l|l|l|l|l|}
\hline Rank & First Name & \multicolumn{2}{l|}{ Last Name } & \multicolumn{2}{l|}{ Title } \\
\hline & \multicolumn{5}{|l|}{ Director of Public Works } \\
\hline Address & & City & State & Postal Code \\
\hline ATTN: AFZN-PW & Fort Riley & KS & $66442-6000$ \\
\hline Work Phone & Ext. & Fax Number & E-Mail Address & \\
\hline & & & & \\
\end{tabular}




\begin{tabular}{|c|c|c|c|}
\hline Facility ID & 18 & Boiler Descriptions & Water tube \\
\hline Facility Name & $\begin{array}{l}\text { U.S. Military } \\
\text { Academy West Point }\end{array}$ & Boiler Serial No. & \\
\hline Boiler Type & 2 boilers & Date Boiler Installed & \\
\hline Other Type & & LNB Install Date & $11 / 1 / 99$ \\
\hline Boiler Size & $150 \mathrm{Mbtu} / \mathrm{hr}$ & LNB Vendor & \\
\hline Boiler Fuel & \#5 Fuel Oil & Date of Major Mod & \\
\hline Combination Fuels & & LNB Type & \\
\hline Burner System & Other & NOx Emission Limit & 0 \\
\hline Other Burner System & & Flame Scanner? & No \\
\hline No. of Burners & 4 each & Flame Scanner Vendor & \\
\hline Boiler Vendor & Keeler & Permit Requirements & No. 5 fuel is 0.30 and Natural Gas is $0.1 \mathrm{lb} / \mathrm{Mbtu}$ \\
\hline \multicolumn{4}{|c|}{ Description of other Modifications } \\
\hline \multicolumn{4}{|c|}{ Description of Commissioning Problems } \\
\hline \multicolumn{4}{|c|}{ Description of Commissioning Solutions } \\
\hline \multicolumn{4}{|c|}{ Description of Installation Problems } \\
\hline \multicolumn{4}{|c|}{$\begin{array}{l}\text { We currently meet NOx RACT by fuel switching at our Central Power Plant (This plant consists of the two largest boilers and the next one } \\
\text { down in size). NOx levels exceed limits. }\end{array}$} \\
\hline \multicolumn{4}{|c|}{ Description of Installation Solutions } \\
\hline \multicolumn{4}{|c|}{$\begin{array}{l}\text { COEN believes that using different burner tips which shape the flame differently will reduce the NOx emissions to a level where fuel } \\
\text { switching is no longer required (I.e., } 0.30 \mathrm{lbs} / \text { million Btu or less). }\end{array}$} \\
\hline \multicolumn{4}{|c|}{ Description of Operational Problems } \\
\hline \multicolumn{4}{|c|}{ Description of Operational Solutions } \\
\hline \multicolumn{4}{|c|}{$\begin{array}{l}\text { We cannot run the two largest boilers and full capacity right now and could not complete the testing of various tips supplied by COEN. We } \\
\text { will be able to resume that testing in January 1999. At low fire, in November of 1998, we were able to (see below) }\end{array}$} \\
\hline \multicolumn{4}{|l|}{ Other Comments } \\
\hline $\begin{array}{l}\text { Achieve[d] } 0.18 \mathrm{lbs} / \mathrm{MBtu} \text { in } \\
\text { meeting standards. }\end{array}$ & e breaching, but not ye & ested at the stack and through 12 & or 15 turndowns. Other boilers appear to be \\
\hline
\end{tabular}

\begin{tabular}{|c|c|c|c|c|c|c|c|}
\hline Rank & \multicolumn{2}{|c|}{ First Name } & Last Name & & \multicolumn{3}{|l|}{ Title } \\
\hline & & & & & \multicolumn{3}{|c|}{ Environmental Group } \\
\hline \multicolumn{4}{|c|}{ Address } & \multicolumn{2}{|l|}{ City } & State & Postal Code \\
\hline \multicolumn{4}{|c|}{ USMA West Point } & \multicolumn{2}{|l|}{ West Point } & NY & 10996-1592 \\
\hline \multicolumn{2}{|c|}{ Work Phone } & Ext. & Fax Number & \multicolumn{4}{|c|}{ E-Mail Address } \\
\hline
\end{tabular}




\begin{tabular}{|c|c|c|c|}
\hline Facility ID & 19 & Boiler Descriptions & Water tube \\
\hline Facility Name & $\begin{array}{l}\text { U.S. Military } \\
\text { Academy West Point }\end{array}$ & Boiler Serial No. & \\
\hline Boiler Type & Other- 3 boilers & Date Boiler Installed & \\
\hline Other Type & & LNB Install Date & \\
\hline Boiler Size & 50 to $150 \mathrm{Mbtu} / \mathrm{hr}$ & LNB Vendor & \\
\hline Boiler Fuel & No. 5 Oil & Date of Major Mod & \\
\hline Combination Fuels & $\begin{array}{l}\text { No. } 5 \text { Oil/Natural } \\
\text { Gas }\end{array}$ & LNB Type & None \\
\hline Burner System & & NOx Emission Limit & 0 \\
\hline Other Burner System & & Flame Scanner? & No \\
\hline No. of Burners & 1 & Flame Scanner Vendor & \\
\hline Boiler Vendor & Tampella; Bigelow & Permit Requirements & No. 5 fuel oil is 0.30 and Natural Gs is 0.10 \\
\hline \multicolumn{4}{|c|}{ Description of other Modifications } \\
\hline \multicolumn{4}{|c|}{ Description of Commissioning Problems } \\
\hline \multicolumn{4}{|c|}{ Description of Commissioning Solutions } \\
\hline \multicolumn{4}{|c|}{ Description of Installation Problems } \\
\hline \multicolumn{4}{|c|}{ Description of Installation Solutions } \\
\hline \multicolumn{4}{|c|}{ Description of Operational Problems } \\
\hline \multicolumn{4}{|c|}{ Description of Operational Solutions } \\
\hline Other Comments & & & \\
\hline
\end{tabular}

\begin{tabular}{|c|c|c|c|c|c|c|c|}
\hline Rank & \multicolumn{2}{|c|}{ First Name } & Last Name & & \multicolumn{3}{|l|}{ Title } \\
\hline & & & & & \multicolumn{3}{|c|}{ Environmental Group } \\
\hline \multicolumn{4}{|c|}{ Address } & \multicolumn{2}{|l|}{ City } & State & Postal Code \\
\hline \multicolumn{4}{|c|}{ USMA West Point } & \multicolumn{2}{|l|}{ West Point } & NY & 10996-1592 \\
\hline \multicolumn{2}{|c|}{ Work Phone } & Ext. & Fax Number & \multicolumn{4}{|c|}{ E-Mail Address } \\
\hline
\end{tabular}




\begin{tabular}{|c|c|c|c|}
\hline Facility ID & 48 & Boiler Descriptions & \\
\hline Facility Name & Andrews AFB & Boiler Serial No. & \\
\hline Boiler Type & 3 new Dual Fuel & Date Boiler Installed & $7 / 1998$ \\
\hline Other Type & & LNB Install Date & 07/13/98 \\
\hline Boiler Size & $85.4 \mathrm{mbtu} / \mathrm{hr}$ & LNB Vendor & Forney Corporation \\
\hline Boiler Fuel & Natural Gas & Date of Major Mod & \\
\hline Combination Fuels & \#6 Fuel Oil & LNB Type & \\
\hline Burner System & & NOx Emission Limit & $.10 \mathrm{lbs} / \mathrm{Mbtu}$ \\
\hline Other Burner System & & Flame Scanner? & No \\
\hline No. of Burners & & Flame Scanner Vendor & \\
\hline Boiler Vendor & English & Permit Requirements & \\
\hline \multicolumn{4}{|c|}{ Description of other Modifications } \\
\hline \multicolumn{4}{|c|}{ Description of Commissioning Problems } \\
\hline \multicolumn{4}{|c|}{ Description of Commissioning Solutions } \\
\hline \multicolumn{4}{|c|}{ Description of Installation Problems } \\
\hline \multicolumn{4}{|c|}{ Description of Installation Solutions } \\
\hline \multicolumn{4}{|c|}{ Description of Operational Problems } \\
\hline \multicolumn{4}{|c|}{ Getting the burners to perform as specified (reach NOx limit) with natural gas } \\
\hline \multicolumn{4}{|c|}{ Description of Operational Solutions } \\
\hline \multicolumn{4}{|c|}{ Burners were field modified; each is now custom and can't be replaced } \\
\hline \multicolumn{4}{|l|}{ Other Comments } \\
\hline
\end{tabular}

\begin{tabular}{|c|c|c|c|c|c|c|}
\hline Rank & \multicolumn{2}{|c|}{ First Name } & Last Name & & \multicolumn{2}{|c|}{ Title } \\
\hline & & & & & \multicolumn{2}{|c|}{ Mechanical Engineer } \\
\hline \multicolumn{4}{|c|}{ Address } & City & State & Postal Code \\
\hline \multicolumn{4}{|c|}{89 CES/CEOE, B3446 Tennessee Ave. } & Andrews AFB & MD & $20762-4803$ \\
\hline \multicolumn{2}{|c|}{ Work Phone } & Ext. & Fax Number & \multicolumn{3}{|c|}{ E-Mail Address } \\
\hline
\end{tabular}




\begin{tabular}{|c|c|c|c|}
\hline Facility ID & 1 & Boiler Descriptions & Cast Iron Section \\
\hline Facility Name & Brooks AFB & Boiler Serial No. & F92-833 \\
\hline Boiler Type & Hot Water, Heating & Date Boiler Installed & March 1993 \\
\hline Other Type & & LNB Install Date & \\
\hline Boiler Size & $560,000 \mathrm{Mbtu} / \mathrm{hr}$ & LNB Vendor & \\
\hline Boiler Fuel & Natural Gas & Date of Major Mod & \\
\hline Combination Fuels & & LNB Type & \\
\hline Burner System & Other & NOx Emission Limit & \\
\hline Other Burner System & $\begin{array}{l}\text { Power Flame, Model } \\
\text { JR30A } 10 \mathrm{HBS}+6\end{array}$ & Flame Scanner? & Yes \\
\hline No. of Burners & 1 & Flame Scanner Vendor & Fire Eye \\
\hline Boiler Vendor & HB Smith & Permit Requirements & None required. \\
\hline \multicolumn{4}{|c|}{ Description of other Modifications } \\
\hline \multicolumn{4}{|c|}{ Description of Commissioning Problems } \\
\hline \multicolumn{4}{|l|}{ None } \\
\hline \multicolumn{4}{|c|}{ Description of Commissioning Solutions } \\
\hline \multicolumn{4}{|c|}{ Description of Installation Problems } \\
\hline \multicolumn{4}{|l|}{ None, contractor installed } \\
\hline \multicolumn{4}{|c|}{ Description of Installation Solutions } \\
\hline \multicolumn{4}{|c|}{ Description of Operational Problems } \\
\hline \multicolumn{4}{|l|}{ None } \\
\hline \multicolumn{4}{|c|}{ Description of Operational Solutions } \\
\hline Other Comments & & & \\
\hline
\end{tabular}

\begin{tabular}{|c|c|c|c|c|c|c|c|}
\hline Rank & \multicolumn{2}{|c|}{ First Name } & Last Name & & \multicolumn{3}{|l|}{ Title } \\
\hline & & & & & \multicolumn{3}{|c|}{ Superintendent of HVAC } \\
\hline \multicolumn{4}{|c|}{ Address } & \multicolumn{2}{|l|}{ City } & State & Postal Code \\
\hline \multicolumn{4}{|c|}{ Brooks AFB } & \multicolumn{2}{|l|}{ San Antonio } & $\mathrm{TX}$ & \\
\hline \multicolumn{2}{|c|}{ Work Phone } & Ext. & Fax Number & \multicolumn{4}{|c|}{ E-Mail Address } \\
\hline
\end{tabular}




\begin{tabular}{|c|c|c|c|}
\hline Facility ID & 33 & Boiler Descriptions & \\
\hline Facility Name & McGuire AFB & Boiler Serial No. & \\
\hline Boiler Type & $\begin{array}{l}3 \text { boilers now being } \\
\text { retrofitted }\end{array}$ & Date Boiler Installed & \\
\hline Other Type & & LNB Install Date & \\
\hline Boiler Size & $61.8 \mathrm{Mbtu} / \mathrm{hr}$ & LNB Vendor & \\
\hline Boiler Fuel & & Date of Major Mod & \\
\hline Combination Fuels & & LNB Type & \\
\hline Burner System & & NOx Emission Limit & 0 \\
\hline Other Burner System & & Flame Scanner? & No \\
\hline No. of Burners & 0 & Flame Scanner Vendor & \\
\hline Boiler Vendor & & Permit Requirements & $\begin{array}{l}\text { In September 1998, McGuire began a project to } \\
\text { renovate/rebuild three large boilers ( } 61.8 \\
\text { MMBtu/hr) with low NOx burners. The estimated } \\
\text { completion date for these boilers is December } \\
1999 .\end{array}$ \\
\hline \multicolumn{4}{|c|}{ Description of other Modifications } \\
\hline \multicolumn{4}{|c|}{ Description of Commissioning Problems } \\
\hline \multicolumn{4}{|c|}{ Description of Commissioning Solutions } \\
\hline \multicolumn{4}{|c|}{ Description of Installation Problems } \\
\hline \multicolumn{4}{|c|}{ Installation is just beginning (September 1998) and will be completed December 1999} \\
\hline \multicolumn{4}{|c|}{ Description of Installation Solutions } \\
\hline \multicolumn{4}{|c|}{ Description of Operational Problems } \\
\hline \multicolumn{4}{|c|}{ Description of Operational Solutions } \\
\hline Other Comments & & & \\
\hline
\end{tabular}

\begin{tabular}{|c|c|c|c|c|c|c|c|}
\hline Rank & \multicolumn{2}{|c|}{ First Name } & Last Name & & \multicolumn{3}{|l|}{ Title } \\
\hline & & & & & \multicolumn{3}{|c|}{ Chief of Infrastructure } \\
\hline \multicolumn{4}{|c|}{ Address } & \multicolumn{2}{|l|}{ City } & State & Postal Code \\
\hline \multicolumn{4}{|c|}{ McGuire AFB } & \multicolumn{2}{|l|}{ Wrightstown } & $\mathrm{NJ}$ & \\
\hline \multicolumn{2}{|c|}{ Work Phone } & Ext. & Fax Number & \multicolumn{4}{|c|}{ E-Mail Address } \\
\hline
\end{tabular}




\begin{tabular}{|c|c|c|c|}
\hline Facility ID & 44 & Boiler Descriptions & fire tube boiler \\
\hline Facility Name & Vandenburg AFB & Boiler Serial No. & \\
\hline Boiler Type & Fire Tube & Date Boiler Installed & 1994 \\
\hline Other Type & & LNB Install Date & 09/03/98 \\
\hline Boiler Size & $2.05 \mathrm{MBtu} / \mathrm{hr}$ & LNB Vendor & S.T. Johnson \\
\hline Boiler Fuel & Propane & Date of Major Mod & \\
\hline Combination Fuels & & LNB Type & $\begin{array}{l}\text { Fully modulating diffuser head forced draft } \\
\text { burner }\end{array}$ \\
\hline Burner System & & NOx Emission Limit & \\
\hline Other Burner System & & Flame Scanner? & No \\
\hline No. of Burners & 1 & Flame Scanner Vendor & \\
\hline Boiler Vendor & Kewanee & Permit Requirements & \\
\hline \multicolumn{4}{|c|}{ Description of other Modifications } \\
\hline \multicolumn{4}{|c|}{ Description of Commissioning Problems } \\
\hline \multicolumn{4}{|c|}{ Description of Commissioning Solutions } \\
\hline \multicolumn{4}{|c|}{ Description of Installation Problems } \\
\hline \multicolumn{4}{|c|}{ Description of Installation Solutions } \\
\hline \multicolumn{4}{|c|}{ Description of Operational Problems } \\
\hline \multicolumn{4}{|c|}{ Description of Operational Solutions } \\
\hline Other Comments & & & \\
\hline
\end{tabular}

\begin{tabular}{|c|c|c|c|c|c|c|c|}
\hline Rank & \multicolumn{2}{|c|}{ First Name } & Last Name & & \multicolumn{3}{|l|}{ Title } \\
\hline & & & & & Engineer & & \\
\hline \multicolumn{4}{|c|}{ Address } & \multicolumn{2}{|l|}{ City } & State & Postal Code \\
\hline \multicolumn{4}{|c|}{ Vandenburg AFB } & \multicolumn{2}{|l|}{ Lompoc } & $\mathrm{CA}$ & \\
\hline \multicolumn{2}{|c|}{ Work Phone } & Ext. & Fax Number & \multicolumn{4}{|c|}{ E-Mail Address } \\
\hline
\end{tabular}




\begin{tabular}{|c|c|c|c|}
\hline Facility ID & 45 & Boiler Descriptions & Model HPG-2500 low-pressure steam boiler \\
\hline Facility Name & Vandenburg AFB & Boiler Serial No. & \\
\hline Boiler Type & $\begin{array}{l}\text { Low-pressure steam } \\
\text { boiler }\end{array}$ & Date Boiler Installed & $12 / 31 / 98$ \\
\hline Other Type & & LNB Install Date & $12 / 31 / 98$ \\
\hline Boiler Size & $2.5 \mathrm{Mbtu} / \mathrm{hr}$ & LNB Vendor & Ajax \\
\hline Boiler Fuel & Natural Gas & Date of Major Mod & \\
\hline Combination Fuels & & LNB Type & premix manifold \\
\hline Burner System & & NOx Emission Limit & \\
\hline Other Burner System & & Flame Scanner? & No \\
\hline No. of Burners & 1 & Flame Scanner Vendor & \\
\hline Boiler Vendor & Ajax & Permit Requirements & \\
\hline \multicolumn{4}{|c|}{ Description of other Modifications } \\
\hline \multicolumn{4}{|c|}{ Description of Commissioning Problems } \\
\hline \multicolumn{4}{|c|}{ Description of Commissioning Solutions } \\
\hline \multicolumn{4}{|c|}{ Description of Installation Problems } \\
\hline \multicolumn{4}{|c|}{ Description of Installation Solutions } \\
\hline \multicolumn{4}{|c|}{ Description of Operational Problems } \\
\hline \multicolumn{4}{|c|}{ Description of Operational Solutions } \\
\hline Other Comments & & & \\
\hline
\end{tabular}

\begin{tabular}{|c|c|c|c|c|c|c|c|}
\hline Rank & \multicolumn{2}{|c|}{ First Name } & Last Name & & \multicolumn{3}{|l|}{ Title } \\
\hline & & & & & Engineer & & \\
\hline \multicolumn{4}{|c|}{ Address } & \multicolumn{2}{|l|}{ City } & State & Postal Code \\
\hline \multicolumn{4}{|c|}{ Vandenburg AFB } & \multicolumn{2}{|l|}{ Lompoc } & $\mathrm{CA}$ & \\
\hline \multicolumn{2}{|c|}{ Work Phone } & Ext. & Fax Number & \multicolumn{4}{|c|}{ E-Mail Address } \\
\hline
\end{tabular}




\begin{tabular}{|c|c|c|c|}
\hline Facility ID & 46 & Boiler Descriptions & New water tube boiler \\
\hline Facility Name & Vandenburg AFB & Boiler Serial No. & \\
\hline Boiler Type & Water Tube & Date Boiler Installed & $3 / 18 / 98$ \\
\hline Other Type & & LNB Install Date & $03 / 18 / 98$ \\
\hline Boiler Size & $2.5 \mathrm{MBtu} / \mathrm{hr}$ & LNB Vendor & Alzeta Corporation \\
\hline Boiler Fuel & Natural Gas & Date of Major Mod & \\
\hline Combination Fuels & & LNB Type & Pyromat Radiation Stabilized power burner \\
\hline Burner System & & NOx Emission Limit & \\
\hline Other Burner System & & Flame Scanner? & No \\
\hline No. of Burners & 1 & Flame Scanner Vendor & \\
\hline Boiler Vendor & PVI Industries, Inc. & Permit Requirements & \\
\hline \multicolumn{4}{|c|}{ Description of other Modifications } \\
\hline \multicolumn{4}{|c|}{ Description of Commissioning Problems } \\
\hline \multicolumn{4}{|c|}{ 1) Inconsistent gas line pressure caused flameout; 2) Clogged heat exchanger in old hot water tank } \\
\hline \multicolumn{4}{|c|}{ Description of Commissioning Solutions } \\
\hline \multicolumn{4}{|c|}{ 1) Restricted the gas line by resizing the orifice in the pressure regulator; 2) Removed and cleaned heat exchanger } \\
\hline \multicolumn{4}{|c|}{ Description of Installation Problems } \\
\hline \multicolumn{4}{|c|}{ Description of Installation Solutions } \\
\hline \multicolumn{4}{|c|}{ Description of Operational Problems } \\
\hline \multicolumn{4}{|c|}{ The electrodes wore out prematurely due to cyclical loading on the boiler causing it to turn on and off. } \\
\hline \multicolumn{4}{|c|}{ Description of Operational Solutions } \\
\hline \multicolumn{4}{|c|}{ It was determined that the boiler was oversized for the application, so the burner will be replaced with a smaller unit. } \\
\hline Other Comments & & & \\
\hline
\end{tabular}

\begin{tabular}{|c|c|c|c|c|c|c|c|}
\hline Rank & \multicolumn{2}{|c|}{ First Name } & Last Name & & \multicolumn{3}{|l|}{ Title } \\
\hline & & & & & \multicolumn{3}{|l|}{ Engineer } \\
\hline \multicolumn{4}{|c|}{ Address } & \multicolumn{2}{|l|}{ City } & State & Postal Code \\
\hline \multicolumn{4}{|c|}{ Vandenburg AFB } & \multicolumn{2}{|l|}{ Lompoc } & $\mathrm{CA}$ & \\
\hline \multicolumn{2}{|c|}{ Work Phone } & Ext. & Fax Number & \multicolumn{4}{|c|}{ E-Mail Address } \\
\hline
\end{tabular}




\begin{tabular}{|c|c|c|c|}
\hline Facility ID & 16 & Boiler Descriptions & Water Tube \\
\hline Facility Name & $\begin{array}{l}\text { Naval District } \\
\text { Washington DC }\end{array}$ & Boiler Serial No. & D2898 \& D2900 \\
\hline Boiler Type & Two Boilers & Date Boiler Installed & $4 / 93$ \\
\hline Other Type & & LNB Install Date & $4 / 1 / 93$ \\
\hline Boiler Size & $85,000 \mathrm{lbs} / \mathrm{hr}$ & LNB Vendor & Peabody \\
\hline Boiler Fuel & Natural Gas & Date of Major Mod & \\
\hline Combination Fuels & \#2 fuel oil & LNB Type & ISC-23 Low NOx FlexiPak, \\
\hline Burner System & $\begin{array}{l}\text { Preferred Rimcor } \\
\text { PCII }\end{array}$ & NOx Emission Limit & $0.1 \mathrm{lb} / \mathrm{Mbtu}$ \\
\hline Other Burner System & $\begin{array}{l}\text { Master Controller } \\
\text { run by Allen-Bradley } \\
\text { PLC rack }\end{array}$ & Flame Scanner? & Yes \\
\hline No. of Burners & 1 & Flame Scanner Vendor & Peabody \\
\hline Boiler Vendor & Nebraska & Permit Requirements & .1 - natural gas, 0.2 - fuel oil lb/Mbtu \\
\hline \multicolumn{4}{|c|}{ Description of other Modifications } \\
\hline \multicolumn{4}{|c|}{ Description of Commissioning Problems } \\
\hline \multicolumn{4}{|c|}{ Problems in lighting and maintaining the fire of the boilers (especially on oil). } \\
\hline \multicolumn{4}{|c|}{ Description of Commissioning Solutions } \\
\hline \multicolumn{4}{|c|}{ Description of Installation Problems } \\
\hline \multicolumn{4}{|c|}{$\begin{array}{l}\text { The main problem was the boilers were improperly designed to operate through a common stack. Also poor flame scanners, linkage } \\
\text { kinematics for the forced draft fan. Incorrect positioning of gas spuds and incorrect oil tips and plugs. }\end{array}$} \\
\hline \multicolumn{4}{|c|}{ Description of Installation Solutions } \\
\hline \multicolumn{4}{|c|}{$\begin{array}{l}\text { The boilers required installation of stack dampers not originally designed to control the lighting and maintaining flame. The linkages for the } \\
\text { forced draft fans were removed because of repeated broken rods. Replaced gas spuds settings and oil tips. }\end{array}$} \\
\hline \multicolumn{4}{|c|}{ Description of Operational Problems } \\
\hline \multicolumn{4}{|c|}{$\begin{array}{l}\text { At present, we have one operational problem, excessive vibration above } 55,000 \mathrm{lb} / \mathrm{hr} \text { on both boilers. It has not been determined whether } \\
\text { the problem is because of the LNB or other equipment. }\end{array}$} \\
\hline \multicolumn{4}{|c|}{ Description of Operational Solutions } \\
\hline \multicolumn{4}{|c|}{$\begin{array}{l}\text { We have tried to install plates in the boiler breaching to reduce vortices shown by one study. Yet, this did not work. Another study will be } \\
\text { conducted by a difference vendor within the next few months to resolve this issue. }\end{array}$} \\
\hline \multicolumn{4}{|l|}{ Other Comments } \\
\hline $\begin{array}{l}\text { Also, flame scanners are e } \\
\text { scanner to view the flame. }\end{array}$ & $\begin{array}{l}\text { emely sensitive with lim } \\
\text { Janner aiming is still dif }\end{array}$ & $\begin{array}{l}\text { ed scope due to the burner throat } \\
\text { ult if moved. }\end{array}$ & onfiguration, so there were problems aiming the \\
\hline
\end{tabular}

\begin{tabular}{|c|c|c|c|c|c|c|c|}
\hline Rank & \multicolumn{2}{|c|}{ First Name } & Last Name & & \multicolumn{3}{|l|}{ Title } \\
\hline & & & & & \multicolumn{3}{|c|}{ Mechanical Engineer } \\
\hline \multicolumn{4}{|c|}{ Address } & \multicolumn{2}{|l|}{ City } & State & Postal Code \\
\hline & & & & \multicolumn{2}{|l|}{ Washington } & $\mathrm{DC}$ & \\
\hline \multicolumn{2}{|c|}{ Work Phone } & Ext. & Fax Number & \multicolumn{4}{|c|}{ E-Mail Address } \\
\hline
\end{tabular}




\begin{tabular}{|c|c|c|c|}
\hline Facility ID & 23 & Boiler Descriptions & $\begin{array}{l}\text { Water tube, field __, side burner in_, original } \\
\text { coal fired }\end{array}$ \\
\hline Facility Name & $\begin{array}{l}\text { Naval Station } \\
\text { Everett }\end{array}$ & Boiler Serial No. & \\
\hline Boiler Type & Water Tube & Date Boiler Installed & \\
\hline Other Type & & LNB Install Date & $1 / 1 / 98$ \\
\hline Boiler Size & 50000 & LNB Vendor & S.T. Johnson \\
\hline Boiler Fuel & Natural Gas & Date of Major Mod & $1 / 22 / 98$ \\
\hline Combination Fuels & & LNB Type & 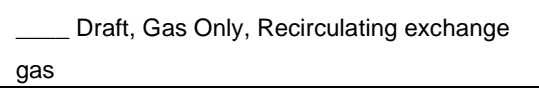 \\
\hline Burner System & Automatic flame & NOx Emission Limit & 0 \\
\hline Other Burner System & Same & Flame Scanner? & Yes \\
\hline No. of Burners & 2 & Flame Scanner Vendor & Fireye \\
\hline Boiler Vendor & Keeler & Permit Requirements & $\begin{array}{l}\text { Air permit applications submitted to NJ Dep, Apri } \\
1998 \text { still } \\
0.1 \mathrm{lb} / \mathrm{hr} \text { Nox Limit will be required to meet the } \\
\text { stack test }\end{array}$ \\
\hline \multicolumn{4}{|c|}{ Description of other Modifications } \\
\hline \multicolumn{4}{|c|}{ Description of Commissioning Problems } \\
\hline \multicolumn{4}{|l|}{ None } \\
\hline \multicolumn{4}{|c|}{ Description of Commissioning Solutions } \\
\hline \multicolumn{4}{|c|}{ Description of Installation Problems } \\
\hline \multicolumn{4}{|c|}{ No major problems (significant) } \\
\hline \multicolumn{4}{|c|}{ Description of Installation Solutions } \\
\hline \multicolumn{4}{|c|}{ Description of Operational Problems } \\
\hline \multicolumn{4}{|l|}{ None } \\
\hline \multicolumn{4}{|c|}{ Description of Operational Solutions } \\
\hline \multicolumn{4}{|l|}{ Other Comments } \\
\hline No & & & \\
\hline
\end{tabular}

\begin{tabular}{|c|c|c|c|c|c|c|c|}
\hline Rank & \multicolumn{2}{|c|}{ First Name } & Last Name & & \multicolumn{3}{|l|}{ Title } \\
\hline & & & & & \multicolumn{3}{|c|}{ Energy Manager } \\
\hline \multicolumn{4}{|c|}{ Address } & \multicolumn{2}{|l|}{ City } & State & Postal Code \\
\hline \multicolumn{4}{|c|}{ Naval Station Everett } & \multicolumn{2}{|l|}{ Everett } & WA & \\
\hline \multicolumn{2}{|c|}{ Work Phone } & Ext. & Fax Number & \multicolumn{4}{|c|}{ E-Mail Address } \\
\hline
\end{tabular}




\begin{tabular}{|c|c|c|c|}
\hline Facility ID & 11 & Boiler Descriptions & \\
\hline Facility Name & $\begin{array}{l}\text { Naval Station } \\
\text { Everett }\end{array}$ & Boiler Serial No. & WG 3879, W3805, W3806, W3807 \\
\hline Boiler Type & $\begin{array}{l}4 \text { Water Tube } \\
\text { Boilers }\end{array}$ & Date Boiler Installed & $1 / 1 / 95$ \\
\hline Other Type & & LNB Install Date & \\
\hline Boiler Size & $\begin{array}{l}3 \times 20,000 \mathrm{lb} / \mathrm{hr} \\
1 \times 30,000 \mathrm{lb} / \mathrm{hr}\end{array}$ & LNB Vendor & \\
\hline Boiler Fuel & Natural Gas & Date of Major Mod & \\
\hline Combination Fuels & \#2 Fuel Oil & LNB Type & None \\
\hline Burner System & $\begin{array}{l}\text { Cleaver Brooks Dual } \\
\text { Fuel (Air \& Steam } \\
\text { Atomization) }\end{array}$ & NOx Emission Limit & $0.05 \mathrm{lb} / \mathrm{Mbtu}$ \\
\hline Other Burner System & & Flame Scanner? & No \\
\hline No. of Burners & 1 & Flame Scanner Vendor & \\
\hline Boiler Vendor & Cleaver Brooks & Permit Requirements & $\begin{array}{l}\text { Plant Design Specs- } 400 \text { ppm CO; } 30 \text { ppm Nox; } \\
80 \text { ppm Sox }\end{array}$ \\
\hline \multicolumn{4}{|c|}{ Description of other Modifications } \\
\hline \multicolumn{4}{|c|}{ Description of Commissioning Problems } \\
\hline \multicolumn{4}{|c|}{ None other than normal tuning curves } \\
\hline \multicolumn{4}{|c|}{ Description of Commissioning Solutions } \\
\hline \multicolumn{4}{|c|}{ Description of Installation Problems } \\
\hline \multicolumn{4}{|l|}{ None } \\
\hline \multicolumn{4}{|c|}{ Description of Installation Solutions } \\
\hline \multicolumn{4}{|c|}{ Description of Operational Problems } \\
\hline \multicolumn{4}{|l|}{ None } \\
\hline \multicolumn{4}{|c|}{ Description of Operational Solutions } \\
\hline \multicolumn{4}{|l|}{ Other Comments } \\
\hline None & & & \\
\hline
\end{tabular}

\begin{tabular}{|c|c|c|c|c|c|c|c|}
\hline Rank & \multicolumn{2}{|c|}{ First Name } & Last Name & & \multicolumn{3}{|l|}{ Title } \\
\hline & & & & & \multicolumn{3}{|c|}{ Energy Manager } \\
\hline \multicolumn{4}{|c|}{ Address } & \multicolumn{2}{|l|}{ City } & State & Postal Code \\
\hline \multicolumn{4}{|c|}{ Naval Station Everett } & \multicolumn{2}{|l|}{ Everett } & WA & \\
\hline \multicolumn{2}{|c|}{ Work Phone } & Ext. & Fax Number & \multicolumn{4}{|c|}{ E-Mail Address } \\
\hline
\end{tabular}




\begin{tabular}{|c|c|c|c|}
\hline Facility ID & 17 & Boiler Descriptions & $\begin{array}{l}\text { Three boilers, two for operation and the third as } \\
\text { a backup }\end{array}$ \\
\hline Facility Name & $\begin{array}{l}\text { Naval Shipyard } \\
\text { Puget Sound }\end{array}$ & Boiler Serial No. & $4024 / 4025 / 4026$ \\
\hline Boiler Type & $\begin{array}{l}\text { Riley VR-C3 (mod) } \\
\text { Balanced Draft ( } 3 \\
\text { boilers) }\end{array}$ & Date Boiler Installed & 1988 \\
\hline Other Type & & LNB Install Date & $8 / 1 / 96$ \\
\hline Boiler Size & $\begin{array}{l}146,444 \mathrm{KBtu} / \mathrm{hr} \text { per } \\
\text { boiler }\end{array}$ & LNB Vendor & COEN \\
\hline Boiler Fuel & No. 2 Oil & Date of Major Mod & $8 / 1 / 96$ \\
\hline Combination Fuels & & LNB Type & CPF/LN-30 \\
\hline Burner System & Coen & NOx Emission Limit & 0.1 \\
\hline Other Burner System & & Flame Scanner? & Yes \\
\hline No. of Burners & 2 per boiler & Flame Scanner Vendor & Fireye \\
\hline Boiler Vendor & Riley & Permit Requirements & Nox Limit: $0.1 \mathrm{lb} / \mathrm{MBtu}$ \\
\hline \multicolumn{4}{|c|}{ Description of other Modifications } \\
\hline \multicolumn{4}{|l|}{ Mod. From coal to natural gas } \\
\hline \multicolumn{4}{|c|}{ Description of Commissioning Problems } \\
\hline \multicolumn{4}{|l|}{ None } \\
\hline \multicolumn{4}{|c|}{ Description of Commissioning Solutions } \\
\hline \multicolumn{4}{|l|}{ None } \\
\hline \multicolumn{4}{|c|}{ Description of Installation Problems } \\
\hline \multicolumn{4}{|l|}{ None } \\
\hline \multicolumn{4}{|c|}{ Description of Installation Solutions } \\
\hline \multicolumn{4}{|l|}{ None } \\
\hline \multicolumn{4}{|c|}{ Description of Operational Problems } \\
\hline \multicolumn{4}{|l|}{02 control } \\
\hline \multicolumn{4}{|c|}{ Description of Operational Solutions } \\
\hline \multicolumn{4}{|c|}{$\begin{array}{l}\text { Previous oil } 02 \text { control was not a concern as it was a backup fuel. It is much more a concern when we switched to natural gas. Existing } \\
\text { forced draft dampers were too course a control system in tune for optimum 02. An } 02 \text { trim system was added. }\end{array}$} \\
\hline \multicolumn{4}{|l|}{ Other Comments } \\
\hline None & & & \\
\hline
\end{tabular}

\begin{tabular}{|c|c|c|c|c|c|c|c|}
\hline Rank & \multicolumn{2}{|c|}{ First Name } & Last Name & & \multicolumn{3}{|l|}{ Title } \\
\hline & & & & & \multicolumn{3}{|c|}{ Mechanical Engineer } \\
\hline \multicolumn{4}{|c|}{ Address } & \multicolumn{2}{|l|}{ City } & State & Postal Code \\
\hline \multicolumn{4}{|c|}{ Naval Shipyard } & \multicolumn{2}{|l|}{ Puget Sound } & WA & \\
\hline \multicolumn{2}{|c|}{ Work Phone } & Ext. & Fax Number & \multicolumn{4}{|c|}{ E-Mail Address } \\
\hline
\end{tabular}




\begin{tabular}{|c|c|c|c|}
\hline Facility ID & 42 & Boiler Descriptions & "SD" Field Ereceted w/ air preheaters \\
\hline Facility Name & $\begin{array}{l}\text { Naval Education and } \\
\text { Training Center }\end{array}$ & Boiler Serial No. & Riley Stoker 3367 \& 3368 \\
\hline Boiler Type & $\begin{array}{l}\text { (2)SD } 2 \text { drum bent } \\
\text { tube front wall-fired }\end{array}$ & Date Boiler Installed & 1959 \\
\hline Other Type & & LNB Install Date & $01 / 01 / 96$ \\
\hline Boiler Size & $75,000 \mathrm{lbs} / \mathrm{hr}$ each & LNB Vendor & DAF \\
\hline Boiler Fuel & $\begin{array}{l}\text { No. } 4 \text { Oil/Natural } \\
\text { Gas }\end{array}$ & Date of Major Mod & \\
\hline Combination Fuels & & LNB Type & Dual fuel, low NOx, induced FGR \\
\hline Burner System & $\begin{array}{l}\text { COEN } 2000 \text { BMS } \\
\text { with PLC (Allen } \\
\text { Bradley) control }\end{array}$ & NOx Emission Limit & \\
\hline Other Burner System & & Flame Scanner? & No \\
\hline No. of Burners & 1 & Flame Scanner Vendor & Fireye \\
\hline Boiler Vendor & Riley Stoker & Permit Requirements & $0.11 \mathrm{lb} / \mathrm{Mbtu}$ \\
\hline \multicolumn{4}{|c|}{ Description of other Modifications } \\
\hline \multicolumn{4}{|c|}{ Description of Commissioning Problems } \\
\hline \multicolumn{4}{|c|}{ Description of Commissioning Solutions } \\
\hline \multicolumn{4}{|c|}{ Description of Installation Problems } \\
\hline \multicolumn{4}{|c|}{ Description of Installation Solutions } \\
\hline \multicolumn{4}{|c|}{ Description of Operational Problems } \\
\hline \multicolumn{4}{|c|}{$\begin{array}{l}\text { 1) Superheat temp high; 2) Improper installation/location of FGR to boiler stack; 3) Hayes controls insufficient for complexity of balanced } \\
\text { draft w/ fgr control. 4) Flame long w/ short fuse box }\end{array}$} \\
\hline \multicolumn{4}{|c|}{ Description of Operational Solutions } \\
\hline \multicolumn{4}{|c|}{$\begin{array}{l}\text { 1) Units were designed for higher temp and pressure, so OK; 2) will relocate tie in to stack; 3) Will replace controls; 4) COEN found the right } \\
\text { tips and flame problems were corrected--Flame is more stable on the older Rileys than the Nebraska boiler. }\end{array}$} \\
\hline \multicolumn{4}{|l|}{ Other Comments } \\
\hline $\begin{array}{l}\text { Stack monitoring } \mathrm{O} 2 \text { a mus } \\
\text { unit) }\end{array}$ & either as alarm or BMS & ut out) and recommend installing & 2 monitoring in the windbox (depending on size of \\
\hline
\end{tabular}

\begin{tabular}{|c|c|c|c|c|c|c|c|}
\hline Rank & \multicolumn{2}{|c|}{ First Name } & \multicolumn{2}{|l|}{ Last Name } & \multicolumn{3}{|l|}{ Title } \\
\hline & & & & & \multicolumn{3}{|c|}{ Supervisor Planning } \\
\hline \multicolumn{4}{|c|}{ Address } & \multicolumn{2}{|l|}{ City } & State & Postal Code \\
\hline \multicolumn{4}{|c|}{$\begin{array}{l}\text { Naval Education and Training Center Public Works Engineering } \\
\text { Division }\end{array}$} & \multicolumn{2}{|l|}{ City } & $\mathrm{RI}$ & \\
\hline \multicolumn{2}{|c|}{ Work Phone } & Ext. & Fax Number & \multicolumn{4}{|c|}{ E-Mail Address } \\
\hline
\end{tabular}




\begin{tabular}{|c|c|c|c|}
\hline Facility ID & 41 & Boiler Descriptions & "D" package type with economizer. \\
\hline Facility Name & $\begin{array}{l}\text { Naval Education and } \\
\text { Training Center }\end{array}$ & Boiler Serial No. & NEB D2092, \\
\hline Boiler Type & $\begin{array}{l}\text { Lea "D" package } \\
\text { type w/economizer }\end{array}$ & Date Boiler Installed & $1 / 1 / 87$ \\
\hline Other Type & & LNB Install Date & $1 / 1 / 94$ \\
\hline Boiler Size & $80,000 \mathrm{lbs} / \mathrm{hr}$ & LNB Vendor & Coen \\
\hline Boiler Fuel & $\begin{array}{l}\text { No. } 4 \text { Oil/Natural } \\
\text { Gas }\end{array}$ & Date of Major Mod & \\
\hline Combination Fuels & & LNB Type & Dual fuel, low NOx, induced FGR \\
\hline Burner System & $\begin{array}{l}\text { COEN } 2000 \text { BMS } \\
\text { with PLC (Allen } \\
\text { Bradley) control }\end{array}$ & NOx Emission Limit & \\
\hline Other Burner System & & Flame Scanner? & Yes \\
\hline No. of Burners & 1 & Flame Scanner Vendor & Coen \\
\hline Boiler Vendor & Nebraska & Permit Requirements & $\begin{array}{l}\text { Nebraska currently the only permitted boiler, } \\
\text { particulate emissions limited to } 0.11 \mathrm{bls} / \mathrm{MMBtu} \\
\text { or } 28.35 \mathrm{lbs} / \mathrm{hr} \text {, whichever is more stringent. } \\
\text { Low sulfur fuel (less than } 0.5 \% \text { ) also required. }\end{array}$ \\
\hline \multicolumn{4}{|c|}{ Description of other Modifications } \\
\hline \multicolumn{4}{|c|}{ Description of Commissioning Problems } \\
\hline \multicolumn{4}{|c|}{ "D" type had problems maintaining low NOx at lower firing rates, FGR not sufficient at the firing rates. } \\
\hline \multicolumn{4}{|c|}{ Description of Commissioning Solutions } \\
\hline \multicolumn{4}{|c|}{$\begin{array}{l}\text { Inlet dampers installed to throttle air opening prior to forced draft fan, thus creating vacuum and or venture effect pulling more FGR in } \\
\text { (obviously the FGR is installed between these new dampers and the forced draft fan. }\end{array}$} \\
\hline \multicolumn{4}{|c|}{ Description of Installation Problems } \\
\hline \multicolumn{4}{|c|}{ No real physical installation problems } \\
\hline \multicolumn{4}{|c|}{ Description of Installation Solutions } \\
\hline \multicolumn{4}{|c|}{ Description of Operational Problems } \\
\hline \multicolumn{4}{|c|}{$\begin{array}{l}\text { 1) Flame instability - "D" type, excessive vibration/ harmonics; 2) insufficient FGR creating higher NOx; 3) FD Fan capacity restriction due to } \\
\text { throttled inlet vanes; 4) Boiler capacity limited due to fan problem; 5) Overspeeding (VFD) fan to compensate }\end{array}$} \\
\hline \multicolumn{4}{|c|}{ Description of Operational Solutions } \\
\hline \multicolumn{4}{|c|}{$\begin{array}{l}\text { 1) Flame stability - Mostly running by COEN for correction but addition of preformatted screen around register helped considerably; 2) } \\
\text { insufficient FGR corrected with the addition of inlet vanes upstream of FG tie in point; 3,4,5) Not resolved }\end{array}$} \\
\hline \multicolumn{4}{|l|}{ Other Comments } \\
\hline
\end{tabular}

\begin{tabular}{|c|c|c|c|c|c|c|c|}
\hline Rank & \multicolumn{2}{|c|}{ First Name } & Last Name & & \multicolumn{3}{|l|}{ Title } \\
\hline & & & & & \multicolumn{3}{|c|}{ Supervisor Planning } \\
\hline \multicolumn{4}{|c|}{ Address } & City & & State & Postal Code \\
\hline \multicolumn{4}{|c|}{ Naval Education and Training Center Public Works Engineering Division } & & & $\mathrm{RI}$ & \\
\hline \multicolumn{2}{|c|}{ Work Phone } & Ext. & Fax Number & \multicolumn{4}{|c|}{ E-Mail Address } \\
\hline
\end{tabular}




\begin{tabular}{|c|c|c|c|}
\hline Facility ID & 20 & Boiler Descriptions & HT Hot Water \\
\hline Facility Name & $\begin{array}{l}\text { Marine Corp Air } \\
\text { Ground Combat } \\
\text { Center }\end{array}$ & Boiler Serial No. & $\# 1$ 14695, \#2 14693, \#3 14694 \\
\hline Boiler Type & Water Tube & Date Boiler Installed & 1977 \\
\hline Other Type & & LNB Install Date & $2 / 1 / 97$ \\
\hline Boiler Size & $40 \mathrm{Mbtu} / \mathrm{hr}$ & LNB Vendor & COEN \\
\hline Boiler Fuel & Natural Gas & Date of Major Mod & \\
\hline Combination Fuels & & LNB Type & Blower Box Extension \\
\hline Burner System & Other & NOx Emission Limit & 0 \\
\hline Other Burner System & Coen & Flame Scanner? & Yes \\
\hline No. of Burners & 3 & Flame Scanner Vendor & \\
\hline Boiler Vendor & I.B.W. & Permit Requirements & $\begin{array}{l}\text { NOx } 70 \text { ppm @ 3\% } 02 \text { and } 0.084 \text { lbs/MBtu } \\
\text { CO } 400 \text { ppm@ 3/6 } 02\end{array}$ \\
\hline \multicolumn{4}{|c|}{ Description of other Modifications } \\
\hline \multicolumn{4}{|c|}{ Description of Commissioning Problems } \\
\hline \multicolumn{4}{|l|}{ None } \\
\hline \multicolumn{4}{|c|}{ Description of Commissioning Solutions } \\
\hline \multicolumn{4}{|c|}{ Description of Installation Problems } \\
\hline \multicolumn{4}{|l|}{ None. } \\
\hline \multicolumn{4}{|c|}{ Description of Installation Solutions } \\
\hline \multicolumn{4}{|l|}{ None } \\
\hline \multicolumn{4}{|c|}{ Description of Operational Problems } \\
\hline \multicolumn{4}{|l|}{ Not low enough NOx } \\
\hline \multicolumn{4}{|c|}{ Description of Operational Solutions } \\
\hline \multicolumn{4}{|l|}{ Stack Air Recirculation } \\
\hline \multicolumn{4}{|l|}{ Other Comments } \\
\hline No & & & \\
\hline
\end{tabular}

\begin{tabular}{|c|c|c|c|c|c|c|c|}
\hline Rank & \multicolumn{2}{|c|}{ First Name } & \multicolumn{2}{|l|}{ Last Name } & \multicolumn{3}{|l|}{ Title } \\
\hline & & & & & \multicolumn{3}{|c|}{ Energy Manager } \\
\hline \multicolumn{4}{|c|}{ Address } & \multicolumn{2}{|l|}{ City } & State & Postal Code \\
\hline \multicolumn{4}{|c|}{ Marine Corp Air Ground Combat Center } & \multicolumn{2}{|l|}{ City } & $\mathrm{CA}$ & \\
\hline \multicolumn{2}{|c|}{ Work Phone } & Ext. & Fax Number & \multicolumn{4}{|c|}{ E-Mail Address } \\
\hline
\end{tabular}




\begin{tabular}{|c|c|c|c|}
\hline Facility ID & 8 & Boiler Descriptions & Firetube \\
\hline Facility Name & $\begin{array}{l}\text { Naval Medical } \\
\text { Center San Diego }\end{array}$ & Boiler Serial No. & $\# 1$ (040903), \#2 (040904), \# (040905) \\
\hline Boiler Type & Multiple & Date Boiler Installed & $1 / 1 / 85$ \\
\hline Other Type & & LNB Install Date & $2 / 1 / 97$ \\
\hline Boiler Size & 16.7 & LNB Vendor & Combustion Specialties \\
\hline Boiler Fuel & Natural Gas & Date of Major Mod & \\
\hline Combination Fuels & $\begin{array}{l}\text { \#2 Diesel Fuel as } \\
\text { Emergency Backup }\end{array}$ & LNB Type & CS-1-17-30 \\
\hline Burner System & Honeywell & NOx Emission Limit & 30 \\
\hline Other Burner System & $\mathrm{N} / \mathrm{A}$ & Flame Scanner? & No \\
\hline No. of Burners & 3 & Flame Scanner Vendor & \\
\hline Boiler Vendor & Superior & Permit Requirements & $\begin{array}{l}\text { NOx Limit on Natural gas 30ppm; NOx Limit on } \\
\text { \#2 Emergency Fuel 40ppm; Co. Limit 300ppm }\end{array}$ \\
\hline \multicolumn{4}{|c|}{ Description of other Modifications } \\
\hline \multicolumn{4}{|c|}{ Description of Commissioning Problems } \\
\hline \multicolumn{4}{|c|}{ No commissioning here at the Hospital, the Damper controls were bad, and the contractor just ignored the problem. } \\
\hline \multicolumn{4}{|c|}{ Description of Commissioning Solutions } \\
\hline \multicolumn{4}{|c|}{ We have started doing our own testing and making adjustments to the system. } \\
\hline \multicolumn{4}{|c|}{ Description of Installation Problems } \\
\hline \multicolumn{4}{|c|}{ Contractor went Bankrupt, this project took over 3 years to complete. } \\
\hline \multicolumn{4}{|c|}{ Description of Installation Solutions } \\
\hline \multicolumn{4}{|c|}{$\begin{array}{l}\text { Get rid of these fly by night Contractor's that rip off the Government on a daily basis. Hold them accountable for the work, if they fail to get } \\
\text { the job done in a timely manner, do not pay them. }\end{array}$} \\
\hline \multicolumn{4}{|c|}{ Description of Operational Problems } \\
\hline \multicolumn{4}{|c|}{ Description of Operational Solutions } \\
\hline \multicolumn{4}{|l|}{ Other Comments } \\
\hline
\end{tabular}

\begin{tabular}{|l|l|l|l|l|l|}
\hline Rank & First Name & \multicolumn{2}{l|}{ Last Name } & \multicolumn{1}{l|}{ Title } \\
\hline & \multicolumn{5}{l|}{ General Foreman } \\
\hline Address & & City & State & Postal Code \\
\hline Naval Medical Center & & San Diego & CA & \\
\hline Work Phone & Ext. & Fax Number & E-Mail Address & \\
\hline & & & & \\
\end{tabular}




\begin{tabular}{|c|c|c|c|}
\hline Facility ID & 6 & Boiler Descriptions & \\
\hline Facility Name & Point Loma & Boiler Serial No. & \#2 (8563-01), \#3 (8563-02) \\
\hline Boiler Type & Two & Date Boiler Installed & $1 / 19 / 98$ \\
\hline Other Type & & LNB Install Date & $01 / 01 / 98$ \\
\hline Boiler Size & 14.7 & LNB Vendor & County Burner \\
\hline Boiler Fuel & Natural Gas & Date of Major Mod & \\
\hline Combination Fuels & & LNB Type & Johnston Burners \\
\hline Burner System & Other & NOx Emission Limit & $30 \mathrm{ppm}$ \\
\hline Other Burner System & & Flame Scanner? & Yes \\
\hline No. of Burners & 2 & Flame Scanner Vendor & Fire Eye \\
\hline Boiler Vendor & Johnston & Permit Requirements & $\begin{array}{ll}\text { Limit Natural Gas } & 30 \mathrm{ppm} \\
\text { CO Limit } & 300 \mathrm{ppm} \\
\end{array}$ \\
\hline \multicolumn{4}{|c|}{ Description of other Modifications } \\
\hline \multicolumn{4}{|c|}{ Description of Commissioning Problems } \\
\hline \multicolumn{4}{|c|}{ Description of Commissioning Solutions } \\
\hline \multicolumn{4}{|c|}{ Description of Installation Problems } \\
\hline \multicolumn{4}{|c|}{ This project went very smooth, no problems to report. Contractor was very professional and extremely knowledgeable } \\
\hline \multicolumn{4}{|c|}{ Description of Installation Solutions } \\
\hline \multicolumn{4}{|c|}{ Description of Operational Problems } \\
\hline \multicolumn{4}{|c|}{ Description of Operational Solutions } \\
\hline Other Comments & & & \\
\hline
\end{tabular}

\begin{tabular}{|c|c|c|c|c|c|c|c|}
\hline \multirow[t]{2}{*}{ Rank } & \multicolumn{2}{|c|}{ First Name } & \multicolumn{2}{|l|}{ Last Name } & \multicolumn{3}{|l|}{ Title } \\
\hline & & & & & \multicolumn{3}{|c|}{ General Foremen } \\
\hline \multicolumn{4}{|c|}{ Address } & \multicolumn{2}{|l|}{ City } & State & Postal Code \\
\hline & & & & \multicolumn{2}{|l|}{ San Diego } & $\mathrm{CA}$ & \\
\hline \multicolumn{2}{|c|}{ Work Phone } & Ext. & Fax Number & \multicolumn{4}{|c|}{ E-Mail Address } \\
\hline
\end{tabular}




\begin{tabular}{|c|c|c|c|}
\hline Facility ID & 7 & Boiler Descriptions & Watertube \\
\hline Facility Name & $\begin{array}{l}\text { Naval Amphloious } \\
\text { Base }\end{array}$ & Boiler Serial No. & \#1 (005729), \#2 (005730) \\
\hline Boiler Type & Multiple & Date Boiler Installed & $1 / 1 / 96$ \\
\hline Other Type & & LNB Install Date & $1 / 1 / 96$ \\
\hline Boiler Size & 20 & LNB Vendor & Coen \\
\hline Boiler Fuel & Natural Gas & Date of Major Mod & \\
\hline Combination Fuels & & LNB Type & Coen Micro NOx Burners \\
\hline Burner System & Other & NOx Emission Limit & $30 \mathrm{ppm}$ \\
\hline Other Burner System & $N / A$ & Flame Scanner? & Yes \\
\hline No. of Burners & 2 & Flame Scanner Vendor & Fire Eye \\
\hline Boiler Vendor & $\begin{array}{l}\text { International Boiler } \\
\text { Works }\end{array}$ & Permit Requirements & $\begin{array}{ll}\text { Limit Natural Gas } & 30 \mathrm{ppm} \\
\text { CO Limit } & 300 \mathrm{ppm}\end{array}$ \\
\hline \multicolumn{4}{|c|}{ Description of other Modifications } \\
\hline \multicolumn{4}{|c|}{ Description of Commissioning Problems } \\
\hline \multicolumn{4}{|c|}{ Trim System did not work properly less than $3 \%$ starving th } \\
\hline \multicolumn{4}{|c|}{ Description of Commissioning Solutions } \\
\hline \multicolumn{4}{|c|}{ We have started doing all the testing and setting ourselves to ensure the quality there, and the job is done first time right. } \\
\hline \multicolumn{4}{|c|}{ Description of Installation Problems } \\
\hline \multicolumn{4}{|c|}{$\begin{array}{l}\text { Contractor went Bankrupt, and didn't complete the job, the Utilities department finished the job, and have brought other contractors in to } \\
\text { help make necessary adjustments and repairs. }\end{array}$} \\
\hline \multicolumn{4}{|c|}{ Description of Installation Solutions } \\
\hline \multicolumn{4}{|c|}{ Description of Operational Problems } \\
\hline \multicolumn{4}{|c|}{ Description of Operational Solutions } \\
\hline Other Comments & & & \\
\hline
\end{tabular}

\begin{tabular}{|c|c|c|c|c|c|c|c|}
\hline Rank & \multicolumn{2}{|c|}{ First Name } & Last Name & & \multicolumn{3}{|l|}{ Title } \\
\hline & & & & & \multicolumn{3}{|c|}{ General Formean } \\
\hline \multicolumn{4}{|c|}{ Address } & \multicolumn{2}{|l|}{ City } & State & Postal Code \\
\hline & & & & \multicolumn{2}{|l|}{ San Diego } & $\mathrm{CA}$ & \\
\hline \multicolumn{2}{|c|}{ Work Phone } & Ext. & Fax Number & \multicolumn{4}{|c|}{ E-Mail Address } \\
\hline
\end{tabular}




\title{
CERL DISTRIBUTION
}

\author{
Chief of Engineers \\ ATTN: CEMP-ET (2) \\ ATTN: CEHEC-IM-LH (2) \\ ATTN: CEHEC-IM-LP (2) \\ ATTN: CECC-R \\ ATTN: CERD-L \\ ATTN: CERD-M \\ Defense Tech Info Center 22304 \\ ATTN: DTIC-O (2)
}


Public reporting burden for this collection of information is estimated to average 1 hour per response, including the time for reviewing instructions, searching existing data sources, gathering and maintaining the data needed, and completing and reviewing the collection of information. Send comments regarding this burden estimate or any other aspect of this collection of Information, including suggestions for reducing this burden, to Washington Headquarters

Services, Directorate for information Operations and Reports, 1215 Jefferson Davis Highway, Suite 1204, Arlington, VA 22202-4302, and to the Office of

\begin{tabular}{|c|c|c|}
\hline 1. AGENCY USE ONLY (Leave Blank) & $\begin{array}{c}\text { 2. REPORT DATE } \\
\text { September } 1999\end{array}$ & $\begin{array}{c}\text { 3. REPORT TYPE AND DATES COVERED } \\
\text { Final }\end{array}$ \\
\hline
\end{tabular}

4. TITLE AND SUBTITLE

5. FUNDING NUMBERS

Survey of Department of Defense Facilities with Low NOx Burners

62784

AT45

6. AUTHOR(S)

UL-XC7

Peter Herz, George Powers, Douglas Uthus, Steven Aylor, and Michael K. Brewer

7. PERFORMING ORGANIZATION NAME(S) AND ADDRESS(ES)

U.S. Army Construction Engineering Research Laboratory (CERL)

P.O. Box 9005

Champaign, IL 61826-9005

8. PEFORMING ORGANIZATION REPORT NUMBER

TR 99/81

9. SPONSORING / MONITORING AGENCY NAME(S) AND ADDRESS(ES)

Headquarters, U.S. Army Corps of Engineers (HQUSACE)

10. SPONSORING / MONITORING

ATTN: CEMP-ET

20 Massachussetts Ave., NW.

Washington, DC 20314-1000

9. SUPPLEMENTARY NOTES

Copies are available from the National Technical Information Service, 5385 Port Royal Road, Springfield, VA 22161

12a. DISTRIBUTION / AVAILABILITY STATEMENT

12b. DISTRIBUTION CODE

Approved for public release; distribution is unlimited.

13. ABSTRACT (Maximum 200 words)

This study conducted a survey regarding recent experience with Low NOx Burners (LNBs) at Department of Defense facilities known to be operating or installing low nitrogen oxides-emitting burners on boilers. The data collected did not conclusively show that LNBs are chronically problematic, but a majority of the LNB-equipped boilers recorded in the data base reported problems with operation, installation, or commissioning. The most frequently reported problems were the failure of the units to reduce NOx emissions to the level promised and flame instability caused by a variety of factors.

\begin{tabular}{|l|l|l|}
\hline $\begin{array}{l}\text { 14. SUBJECT TERMS } \\
\text { Department of Defense } \\
\text { air quality }\end{array}$ & $\begin{array}{l}\text { boilers } \\
\text { Low NOx Burners (LNBs) }\end{array}$ \\
\hline $\begin{array}{l}\text { 17. SECURITY CLASSIFICATION } \\
\text { OF REPORT } \\
\text { Unclassified }\end{array}$ & $\begin{array}{c}\text { 18. SECURITY CLASSIFICATION } \\
\text { OF THIS PAGE } \\
\text { Unclassified }\end{array}$ & $\begin{array}{l}\text { 19. SECURITY CLASSIFICATION } \\
\text { OF ASTRACT } \\
\text { Unclassified }\end{array}$ \\
\hline
\end{tabular}

